\title{
Constrained KP Hierarchy and Bi-Hamiltonian Structures
}

\author{
W. Oevel ${ }^{1}$ and W. Strampp ${ }^{2}$ \\ 1 Dept. of Mathematical Sciences, University of Technology, Loughborough, LE11 3TU, U.K. \\ ${ }^{2}$ FB 17-Mathematik, GH-Universität Kassel, Holländische Str. 36, D-34127 Kassel, Germany
}

Received July 3, 1992

\begin{abstract}
The Kadomtsev-Petviashvili (KP) hierarchy is considered together with the evolutions of eigenfunctions and adjoint eigenfunctions. Constraining the KP flows in terms of squared eigenfunctions one obtains $1+1$-dimensional integrable equations with scattering problems given by pseudo-differential Lax operators. The bi-Hamiltonian nature of these systems is shown by a systematic construction of two general Poisson brackets on the algebra of associated Lax-operators. Gauge transformations provide Miura links to modified equations. These systems are constrained flows of the modified KP hierarchy, for which again a general description of their bi-Hamiltonian nature is given. The gauge transformations are shown to be Poisson maps relating the bi-Hamiltonian structures of the constrained KP hierarchy and the modified KP hierarchy. The simplest realization of this scheme yields the AKNS hierarchy and its Miura link to the Kaup-Broer hierarchy.
\end{abstract}

\section{Contents}

1. Introduction. . . . . . . . . . . . . . . . . . . . . . . . . . . . . . 51

2. General Background and Basic Definitions . . . . . . . . . . . . . . . . 53

3. Constrained KP Flows and Their Bi-Hamiltonian Structure . . . . . . . . . 55

4. Constrained Modified KP Flows and Their Bi-Hamiltonian Structure . . . . . . 64

5. Miura Links and Gauge Transformations as Poisson Maps . . . . . . . . . 68

6. Examples . . . . . . . . . . . . . . . . . . . . . . . . . . . . . . . 69

7. Conclusions and Perspectives . . . . . . . . . . . . . . . . . . . . . . . . . . . . . 78

References . . . . . . . . . . . . . . . . . . . 80

\section{Introduction}

Many finite dimensional integrable systems arise from partial differential equations in soliton theory. Typical reduction schemes from partial to ordinary differential equations involve pole expansions $[1,2]$ or stationary flows $[3]$ and reductions to pure soliton submanifolds [4]. For the latter case a systematic "nonlinearization" procedure was proposed by $\mathrm{Cao}$ [5]. The main idea is that squared eigenfunctions 
(i.e. the products of eigenfunctions $\Phi_{i}$ and adjoint eigenfunctions $\Psi_{i}$ ) associated with the underlying linear scattering problems may be regarded as conserved covariants of the nonlinear hierarchy of integrable evolution equations under consideration. Finite dimensional invariant submanifolds for these flows are given by the stationary points of conserved covariants, hence constraints such as $U=\sum_{i} \Phi_{i} \Psi_{i}$, say, will be preserved under the dynamics, if the potential $U$ is a conserved covariant of the evolution under consideration.

This idea has been generalized to reduce soliton equations in $2+1$ dimensions to simpler integrable partial differential equations in $1+1$ dimensions. In particular, for the Kadomtsev-Petviashvili hierarchy the space-derivative $\left(\sum_{i} \Phi_{i} \Psi_{i}\right)_{x}$ of squared eigenfunctions represents a symmetry generator ([6]). Hence, using any symmetry $U_{t_{\mathrm{N}}}$ of the KP-hierarchy the constraint $U_{t_{N}}=\left(\sum_{i} \Phi_{i} \Psi_{i}\right)_{x}$ is compatible with all $2+1$-dimensional flows of the KP hierarchy, thus leading to a hierarchy of commuting integrable equations in $1+1$ dimensions ([7-12]). In particular, the resulting system for $t_{N}=x$ was shown to represent the (multi-component) AKNS hierarchy, the use of the next higher KP symmetries lead to multicomponent versions of the hierarchies described by Yajima-Oikawa [13] and Melnikov [14].

These reductions were found to have a simple description using Sato's construction ([15-19]) of the KP hierarchy. There, the KP hierarchy is encoded in the Lax equations

$$
\frac{d}{d t_{n}} L_{\mathrm{KP}}=\left[\left(L_{\mathrm{KP}}^{n}\right)_{+}, L_{\mathrm{KP}}\right], \quad n \in \mathbb{N},
$$

for the pseudo-differential Lax operator

$$
L_{\mathrm{KP}}=\partial+U \partial^{-1}+U_{2} \partial^{-2}+U_{3} \partial^{-3}+\ldots,
$$

where $\left(L_{\mathrm{KP}}^{n}\right)_{+}$is the projection of the power $L_{\mathrm{KP}}^{n}$ to its purely differential part. Upon elimination of the "auxiliary" fields $U_{2}, U_{3}, \ldots$ from (1.1) the remaining equations for the prime field $U$ in (1.2) represents for KP equation and its higher flows. In this case eigenfunctions $\Phi$ and adjoint eigenfunctions $\Psi$ are introduced as solutions of the linear problems

$$
\frac{d}{d t_{n}} \Phi=\left(L_{\mathrm{KP}}^{n}\right)_{+} \Phi, \frac{d}{d t_{n}} \Psi=-\left(L_{\mathrm{KP}}^{n}\right)_{+}^{*} \Psi .
$$

It was observed ([20-22]) that the constraint

$$
L_{\mathrm{KP}}^{N}=\left(L_{\mathrm{KP}}^{N}\right)_{+}+\Phi \partial^{-1} \Psi=\partial^{N}+u_{N-2} \partial^{N-2}+\ldots+u_{0}+\Phi \partial^{-1} \Psi=: L
$$

on the coefficients of the operator (1.2) coincides with the symmetry constraint $U_{t_{N}}=(\Phi \Psi)_{x}$ of the nonlinearization approach. All coefficients $U, U_{2}, \ldots$ in (1.2) can be expressed as differential expression of the coefficients $u_{N-2}, \ldots, u_{0}, \Phi, \Psi$ of $L_{\mathrm{KP}}^{N}$ in (1.4), hence they may be regarded as the fields parametrizing (1.2). The equations (1.1) thus reduce to a hierarchy of $1+1$-dimensional equations for the fields $u_{N-2}, \ldots, u_{0}, \Phi$, and $\Psi$. In this set-up the recursion operators and biHamiltonian formulations for some of the simplest 1+1-dimensional reductions of the KP-hierarchy have been identified [21,22].

The aim of this paper is to provide a unified description of the bi-Hamiltonian structure associated with Lax operators $L$ of the form (1.4). The basic tools are 
the two Hamiltonian formulations given by the Lie-Poisson structure of the Adler-Kostant-Symes scheme

$$
\frac{d}{d t} L=\left[(\nabla H)_{+}, L\right]-[\nabla H, L]_{+},
$$

and Gelfand and Dikii's quadratic structure

$$
\frac{d}{d t} L=(L \nabla H)_{+} L-L(\nabla H L)_{+}
$$

originally associated with purely differential operators of the form $L=\partial^{N}+$ $u_{N-2} \partial^{N-2}+\ldots+u_{0}([23])$. A Lie-algebraic setting for this Hamiltonian scenario was given by Adler [24], a more general description in terms of classical $r$-matrices was found by Semenov [25]. It will be shown that the corresponding Poisson brackets can be properly restricted to differential operators supplemented by a pseudo-differential part of the particular form $\Phi \partial^{-1} \Psi$. Hence, the bi-Hamiltonian formulations for the $1+1$-dimensional hierarchies obtained as restrictions (1.4) of the KP hierarchy are simply given by (1.5) and (1.6).

In Sect. 2 we briefly review the essentials of pseudo-differential symbols to be used for the description of the constrained KP hierarchy. In Sect. 3 we establish a lifting of the Hamiltonian formulations (1.5) and (1.6) to a larger space given by operators $L$ together with their eigenfunctions and adjoint eigenfunctions. In the restriction to the constrained situation (1.4), these Poisson structures provide a convenient parametrization of the brackets (1.5) and (1.6). In Sect. 4 an analogous construction is proposed for the modified KP hierarchy. Section 5 is devoted to Miura transformations between the KP hierarchy and the modified KP hierarchy. The Miura transformation is given by a gauge transformation triggered by an eigenfunction of the KP hierarchy. The Hamiltonian structures associated with the constrained KP flows are mapped to the Hamiltonian structures associated with the constrained modified KP by means of this gaugetransformation. To illustrate the general results, the simplest $1+1$-dimensional constraints of the KP and modified KP hierarchies and their bi-Hamiltonian structures are worked out in Sect. 6.

\section{General Background and Basic Definitions}

We consider pseudo-differential symbols of the form

$$
L \in g=\left\{\sum_{n=0}^{N} a_{n} \partial^{n}+\sum_{n<0} \partial^{n} a_{n}\right\}
$$

with coefficients $a_{n}$ which are functions of a "space variable" $x$. In the following we will always identify functions with the multiplication operator given by this function. The space $g$ of all operators (2.1) is endowed with an algebra structure requiring that for positive $n$ the symbol $\partial^{n}$ is the power of the differential operator $\partial=\partial / \partial x$, whereas negative powers are formal integrations defined by the basic rule

$$
\begin{aligned}
& \partial^{-1} a=a \partial^{-1}-a_{x} \partial^{-2}+a_{x x} \partial^{-3} \mp \ldots, \\
& a \partial^{-1}=\partial^{-1} a+\partial^{-2} a_{x}+\partial^{-3} a_{x x}+\ldots
\end{aligned}
$$


We define projections of (2.1) to various differential orders by

$$
P_{n}(L)=\left\{\begin{array}{ll}
a_{n} \partial^{n} & \text { for } n \geqq 0, \\
\partial^{n} a_{n} & \text { for } n<0,
\end{array} \quad P_{\geqq k}(L)=\sum_{n \geqq k} P_{n}(L), \quad P_{<k}(L)=\sum_{n<k} P_{n}(L) .\right.
$$

In [24] Adler noted that the residue of the pseudo-differential symbols gives rise to a trace formalism on this algebra

$$
\operatorname{tr}(L):=\int \operatorname{res}(L) d x, \quad \operatorname{res}(L)=\operatorname{res}\left(\sum_{n \geqq 0} a_{n} \partial^{n}+\sum_{n<0} \partial^{n} a_{n}\right):=a_{-1} .
$$

With $\operatorname{tr}([A, B])=0$ one obtains a symmetric duality bracket

$$
\langle L, \tilde{L}\rangle:=\operatorname{tr}(L \tilde{L})=\langle\tilde{L}, L\rangle
$$

on $g$, so that - applying the Adler-Kostant-Symes scheme [24] - one can immediately construct commuting hierarchies of Hamiltonian Lax equations

$$
\frac{d}{d t} L=\left[P_{\geqq 0}\left(L^{q}\right), L\right]
$$

on $g$. Here $q$ is an integer or fractional power of the Lax operator under consideration labeling the elements of the integrable hierarchy. The choice

$$
L=L_{\mathrm{KP}}=\partial+U \partial^{-1}+U_{2} \partial^{-2}+U_{3} \partial^{-3}+\ldots
$$

with integer powers $q$ in (2.6) leads to the well known construction of the KP hierarchy in Sato's theory $([15-19])$. The choice

$$
L=L_{\mathrm{GD}}=\partial^{N}+u_{N-2} \partial^{N-2}+\ldots+u_{1} \partial+u_{0}
$$

with fractional powers $q$ in (2.6) leads to Gelfand-Dikii's construction [23] of integrable $1+1$-dimensional systems related to the purely differential Lax operators (2.8) of arbitrary order $N$. These equations can be understood as reductions of the KP hierarchy imposing the constraint

$$
\left(L_{\mathrm{KP}}\right)^{N}=L_{\mathrm{GD}}=\text { purely differential, }
$$

from which all fields $U, U_{2}, U_{3}, \ldots$ in (2.7) can be expressed as differential expressions of the $N-1$ fields $u_{N-2}, \ldots, u_{0}$ in (2.8)

Here we are interested in further reductions of the KP hierarchy induced by eigenfunctions and adjoint eigenfunctions of the Lax operators. In particular, we want to identify reductions of the "coupled" system

$$
\frac{d}{d t}\left(\begin{array}{c}
L \\
\Phi \\
\Psi
\end{array}\right)=\left(\begin{array}{c}
{\left[P_{\geqq 0}\left(L^{q}\right), L\right]} \\
P_{0}\left(L^{q} \Phi\right) \\
-P_{0}\left(\left(L^{q}\right)^{*} \Psi\right)
\end{array}\right),
$$

where $L \in g$ is a pseudo-differential operator of the general form (2.1), and $\Phi, \Psi$ are functions. The symbol $*$ is to denote the usual transposition

$$
\left(\sum_{n} a_{n} \partial^{n}\right)^{*}=\sum_{n}(-1)^{n} \partial^{n} a_{n}
$$

of pseudo-differential symbols. 
Here we stress that in order to avoid confusion in our notation we will never apply a differential operator to a function. Instead, we observe that the composition of a differential operator $A=a_{0}+a_{1} \partial+\ldots+a_{N} \partial^{N}$ with the multiplication operator given by a function $\Phi$, say, is of the form

$$
\begin{aligned}
A \Phi= & \left(a_{0}+a_{1} \partial+\cdots+a_{N} \partial^{N}\right) \Phi=\left(a_{0} \Phi+a_{1} \Phi_{x}+\cdots+a_{N} \Phi_{x \ldots x}\right) \\
& +\left(a_{1} \Phi+a_{2} \Phi_{x}+\cdots\right) \partial+\cdots+(\ldots) \partial^{N},
\end{aligned}
$$

so that applying the operator $A$ to the function $\Phi$ can be described as the zero order term $P_{0}(A \Phi)$ of the operator $A \Phi$. Hence, for arbitrary pseudo-differential operator $A$ the symbol $P_{0}(A \Phi)$ may be understood as the function obtained from letting the differential part $P_{\geqq 0}(A)$ act on the function $\Phi$. The evolutions for $\Phi$ and $\Psi$ in (2.10) should be regarded this way. Hence, if $L$ is a purely differential operator, the functions $\Phi$ and $\Psi$ in (2.10) may be understood as eigenfunctions and adjoint eigenfunctions of $L$. In particular, one may impose the scattering equations

$$
P_{0}(L \Phi)=\lambda \Phi, \quad P_{0}\left(L^{*} \Psi\right)=\mu \Psi,
$$

with eigenvalues $\lambda, \mu$ as admissible constraints on the evolution (2.10).

At this stage it seems rather artificial to supplement the evolution for $L$ by the evolutions of $\Phi$ and $\Psi$, as the equation for $L$ does not couple with these additional functions. However, it will turn out that (2.10) admits a reduction to operators of the form

$$
L=\partial^{N}+u_{N-2} \partial^{N-2}+\cdots+u_{0}+\Phi \partial^{-1} \Psi .
$$

Inserting this operator into (2.10) one finds highly non-trivial coupled integrable systems mixing the components $u_{N-2}, \ldots, u_{0}$ of $L$ as well as $\Phi$ and $\Psi$.

Moreover, it turns out that the system (2.10) admits a rather interesting biHamiltonian formulation. These Poisson structures will admit proper restrictions to the manifold of operators (2.14), so that the bi-Hamiltonian formulation for these restricted systems can be obtained from the Hamiltonian formulations of the general system (2.10). Hence, we will construct Poisson brackets for the triples $(L, \Phi, \Psi)$, which are regarded as elements of the space $\tilde{g}=g \oplus \mathscr{H} \oplus \mathscr{H}$ with $\mathscr{H}$ an appropriate Hilbert space from which the (adjoint) eigenfunctions $\Phi$ and $\Psi$ are taken. As duality bracket on $\tilde{g}$ we will use

$$
\langle(L, \Phi, \Psi),(\tilde{L}, \tilde{\Phi}, \tilde{\Psi})\rangle=\operatorname{tr}(L \tilde{L})+\int(\Phi(x) \tilde{\Phi}(x)+\Psi(x) \tilde{\Psi}(x)) d x .
$$

\section{Constrained KP Flows and their Bi-Hamiltonian Structure}

The principal aim of this section is to establish a bi-Hamiltonian formulation for the evolution (2.10), which we then want to restrict to Lax operators of the form (2.14). On the algebra $g$, i.e. just considering the Lax hierarchy $\frac{d}{d t} L=\left[P_{\geqq 0}\left(L^{q}\right), L\right]$, it is well known ([23-25]) that a linear Poisson bracket for these equations is given by the Poisson tensor

$$
\frac{\delta H}{\delta L} \rightarrow\left[P_{\geqq 0}\left(\frac{\delta H}{\delta L}\right), L\right]-P_{\geqq 0}\left(\left[\frac{\delta H}{\delta L}, L\right]\right) .
$$


This is the Lie-Poisson structure of a Lie bracket on $g$ stemming from an algebra decomposition of $g$ (see e.g. [25] for an explanation in terms of classical $r$ matrices). In order to obtain a first Hamiltonian structure for the extended system (2.10) we have to find a lifting of this Poisson structure to the bundle $\tilde{g}=g \oplus \mathscr{H} \oplus \mathscr{H}$ of triplets $(L, \Phi, \Psi)$. The answer is given by

Theorem 1. (The lifted linear bracket). The mapping

$$
\begin{gathered}
\mathscr{P}_{1}(L, \Phi, \Psi):\left(\begin{array}{l}
\frac{\delta H}{\delta L} \\
\frac{\delta H}{\delta \Phi} \\
\frac{\delta H}{\delta \Psi}
\end{array}\right) \\
\rightarrow\left(\begin{array}{c}
{\left[P_{\geqq 0}\left(\frac{\delta H}{\delta L}\right), L\right]-P_{\geqq 0}\left(\left[\frac{\delta H}{\delta L}, L\right]\right)-\Phi \partial^{-1} \frac{\delta H}{\delta \Phi}+\frac{\delta H}{\delta \Psi} \partial^{-1} \Psi} \\
P_{0}\left(\frac{\delta H}{\delta L}\right)^{-1}+\frac{\delta H}{\delta \Psi} \\
-P_{0}\left(\left(\frac{\delta H}{\delta L}\right)^{*} \Psi\right)-\frac{\delta H}{\delta \Phi}
\end{array}\right)
\end{gathered}
$$

defines a Poisson tensor on $\tilde{g}$.

We note that $\frac{\delta H}{\delta L} \in g$ is a pseudo-differential operator, whereas $\frac{\delta H}{\delta \Phi}, \frac{\delta H}{\delta \Psi} \in \mathscr{H}$ are functions. The proof is very simple starting with the tensor

$$
\tilde{\mathscr{P}}_{1}(l, \Phi, \Psi):\left(\begin{array}{c}
\frac{\delta H}{\delta l} \\
\frac{\delta H}{\delta \Phi} \\
\frac{\delta H}{\delta \Psi}
\end{array}\right) \rightarrow\left(\begin{array}{c}
{\left[P_{\geqq 0}\left(\frac{\delta H}{\delta l}\right), l\right]-P_{\geqq 0}\left(\left[\frac{\delta H}{\delta l}, l\right]\right)} \\
\frac{\delta H}{\delta \Psi} \\
-\frac{\delta H}{\delta \Phi}
\end{array}\right),
$$

on $g \oplus \mathscr{H} \oplus \mathscr{H}$ using coordinates $(l, \Phi, \Psi)$. The map (3.3) clearly defines a Poisson structure. Considering the change of coordinates

$$
T:(l, \Phi, \Psi) \rightarrow(L, \Phi, \Psi), \quad L:=l+\Phi \partial^{-1} \Psi
$$

one calculates

$$
T^{\prime}:\left(\begin{array}{l}
A \\
\alpha \\
\beta
\end{array}\right) \rightarrow\left(\begin{array}{c}
A+\alpha \partial^{-1} \Psi+\Phi \partial^{-1} \beta \\
\alpha \\
\beta
\end{array}\right), \quad T^{\dagger}:\left(\begin{array}{l}
A \\
\alpha \\
\beta
\end{array}\right) \rightarrow\left(\begin{array}{c}
A \\
P_{0}\left(A^{*} \Psi\right)+\alpha \\
P_{0}(A \Phi)+\beta
\end{array}\right)
$$

as the linearization $T^{\prime}$ and its transposed $T^{\prime \dagger}$ (w.r.t. the duality (2.15)). Here $A \in g$ is a pseudo-differential operator, whereas $\alpha, \beta \in \mathscr{H}$ are functions. Now a straightforward calculation shows that the image $T^{\prime} \widetilde{\mathscr{P}}_{1} T^{\prime \dagger}$ of (3.3) yields (3.2) in the new 
coordinate system given by $(L, \Phi, \Psi)$. Hence, the Poisson properties of (3.3) are inherited to (3.2).

A systematic construction of a second Poisson bracket associated with the Lax equations (2.6) was given by Gelfand and Dikii [23], a more general setting in terms of classical $r$-matrices was found by Semenov [25]. On $g$ this bracket is given by the quadratic tensor

$$
\frac{\delta H}{\delta L} \rightarrow P_{\geqq 0}\left(L \frac{\delta H}{\delta L}\right) L-L P_{\geqq 0}\left(\frac{\delta H}{\delta L} L\right) .
$$

Having in mind the coupled equations (2.10) we have to search for a lift of this tensor to the bundle $\tilde{g}$. The extended Poisson tensor turns out to be non-local:

Theorem 2. (The lifted quadratic bracket.) The mapping

$$
\begin{array}{r}
\mathscr{P}_{2}(L, \Phi, \Psi):\left(\begin{array}{c}
\frac{\delta H}{\delta L} \\
\frac{\delta H}{\delta \Phi} \\
\frac{\delta H}{\delta \Psi}
\end{array}\right) \\
\rightarrow\left(\begin{array}{c}
P_{\geqq 0}\left(L \frac{\delta H}{\delta L}\right) L-L P_{\geqq 0}\left(\frac{\delta H}{\delta L} L\right)-\Phi \partial^{-1} \frac{\delta H}{\delta \Phi} L+L \frac{\delta H}{\delta \Psi} \partial^{-1} \Psi \\
P_{0}\left(L \frac{\delta H}{\delta L} \Phi\right)-\Phi D^{-1}\left(\Phi \frac{\delta H}{\delta \Phi}\right)+\Phi D^{-1}\left(\Psi \frac{\delta H}{\delta \Psi}\right)+P_{0}\left(L \frac{\delta H}{\delta \Psi}\right) \\
-P_{0}\left(L^{*}\left(\frac{\delta H}{\delta L}\right)^{*} \Psi\right)+\Psi D^{-1}\left(\Phi \frac{\delta H}{\delta \Phi}\right)-P_{0}\left(L^{*} \frac{\delta H}{\delta \Phi}\right)-\Psi D^{-1}\left(\Psi \frac{\delta H}{\delta \Psi}\right)
\end{array}\right)
\end{array}
$$

defines a Poisson tensor on $\tilde{g}$.

Here, $D^{-1}$ defines the integral operation

$$
D^{-1}(f)=\frac{1}{2}\left(\int_{-\infty}^{x} f(\xi) d \xi-\int_{x}^{\infty} f(\xi) d \xi\right),
$$

acting on functions with suitable boundary conditions, which is to be distinguished from the pseudo-differential symbol $\partial^{-1}$ acting on a suitable space of test functions. Unfortunately, the proof is not as simple as the proof of the Poisson properties of the lifted linear bracket. In fact, following the proof of Theorem 1 and mapping $\mathscr{P}_{2}$ to a chart involving $l=L-\Phi \partial^{-1} \Psi$, one finds an image of $\mathscr{P}_{2}$ with an even more complicated form. As we do not see a simple structural argument for the Poisson properties of (3.7) we have checked $\mathscr{P}_{2}$ by a most tiring direct calculation. The details of this cumbersome analysis are omitted.

Theorem 3. The Poisson structures given by (3.2) and (3.7) are compatible.

Fortunately, this proof is very simple using a standard argument. We consider the shift $L \rightarrow \widetilde{L}:=L-1$ resulting in the image

$$
\tilde{\mathscr{P}}_{2}(\tilde{L}, \Phi, \Psi)=\mathscr{P}_{1}(\tilde{L}, \Phi, \Psi)+\mathscr{P}_{2}(\tilde{L}, \Phi, \Psi) .
$$


Hence, $\mathscr{P}_{1}+\mathscr{P}_{2}$ is again a Poisson structure. We observe that $\mathscr{P}_{1}, \mathscr{P}_{2}$ provide Poisson brackets for the coupled equations (2.10):

$$
\begin{aligned}
\frac{d}{d t}\left(\begin{array}{l}
L \\
\Phi \\
\Psi
\end{array}\right) & =\left(\begin{array}{c}
{\left[P_{\geqq 0}\left(L^{q}\right), L\right]} \\
P_{0}\left(L^{q} \Phi\right) \\
-P_{0}\left(\left(L^{q}\right)^{*} \Psi\right)
\end{array}\right) \\
& =\left\{\begin{array}{c}
\mathscr{P}_{1}\left(\begin{array}{c}
L^{q} \\
0 \\
0
\end{array}\right)=\mathscr{P}_{1} \operatorname{grad} \frac{1}{q+1} \operatorname{tr}\left(L^{q+1}\right), \\
\mathscr{P}_{2}\left(\begin{array}{c}
L^{q-1} \\
0 \\
0
\end{array}\right)=\mathscr{P}_{2} \operatorname{grad} \frac{1}{q} \operatorname{tr}\left(L^{q}\right) .
\end{array}\right.
\end{aligned}
$$

Remark 1. One may ignore the evolution of $\Psi$ and obtains the bi-Hamiltonian equations

$$
\frac{d}{d t}\left(\begin{array}{l}
L \\
\Phi
\end{array}\right)=\left(\begin{array}{c}
{\left[P_{\geqq 0}\left(L^{q}\right), L\right]} \\
P_{0}\left(L^{q} \Phi\right)
\end{array}\right)=\left\{\begin{array}{c}
\hat{\mathscr{P}}_{1}\left(\begin{array}{c}
L^{q} \\
0
\end{array}\right)=\hat{\mathscr{P}}_{1} \operatorname{grad} \frac{1}{q+1} \operatorname{tr}\left(L^{q+1}\right) \\
\hat{\mathscr{P}}_{2}\left(\begin{array}{c}
L^{q-1} \\
0
\end{array}\right)=\hat{\mathscr{P}}_{2} \operatorname{grad} \frac{1}{q} \operatorname{tr}\left(L^{q}\right)
\end{array}\right.
$$

Here

$$
\hat{\mathscr{P}}_{1}(L, \Phi):\left(\begin{array}{c}
\frac{\delta H}{\delta L} \\
\frac{\delta H}{\delta \Phi}
\end{array}\right) \rightarrow\left(\begin{array}{c}
{\left[P_{\geqq 0}\left(\frac{\delta H}{\delta L}\right), L\right]-P_{\geqq 0}\left(\left[\frac{\delta H}{\delta L}, L\right]\right)-\Phi \partial^{-1} \frac{\delta H}{\delta \Phi}} \\
P_{0}\left(\frac{\delta H}{\delta L} \Phi\right)
\end{array}\right),
$$

and

$$
\hat{\mathscr{P}}_{2}(L, \Phi):\left(\begin{array}{l}
\frac{\delta H}{\delta L} \\
\frac{\delta H}{\delta \Phi}
\end{array}\right) \rightarrow\left(\begin{array}{c}
P_{\geqq 0}\left(L \frac{\delta H}{\delta L}\right) L-L P_{\geqq 0}\left(\frac{\delta H}{\delta L} L\right)-\Phi \partial^{-1} \frac{\delta H}{\delta \Phi} L \\
P_{0}\left(L \frac{\delta H}{\delta L} \Phi\right)-\Phi D^{-1}\left(\Phi \frac{\delta H}{\delta \Phi}\right)
\end{array}\right)
$$

are the $(L, \Phi)$-blocks of the Poisson tensors (3.2) and (3.7), respectively. As these blocks do not depend on the variable $\Psi$, they may be considered as the image of $\mathscr{P}_{1}$ and $\mathscr{P}_{2}$ under the projection map $(L, \Phi, \Psi) \rightarrow(L, \Phi)$. Hence, (3.12) and (3.13) are compatible Poisson tensors on $g \oplus \mathscr{H}$.

The bi-Hamiltonian equations (3.10) admit interesting reductions, which in fact turn out to be Hamiltonian restrictions to invariant Poisson submanifolds.

Theorem 4. (Restrictions).

i) The linear bracket $\mathscr{P}_{1}$ can be properly restricted to triplets $(L, \Phi, \Psi) \in g \oplus \mathscr{H} \oplus \mathscr{H}$ of the form

$$
L=\partial^{N}+u_{N-2} \partial^{N-2}+\cdots+u_{1} \partial+u_{0}+\Phi \partial^{-1} \Psi, \quad N=1,2, \ldots
$$


ii) The quadratic bracket $\mathscr{P}_{2}$ can be properly restricted to triplets $(L, \Phi, \Psi) \in g \oplus \mathscr{H} \oplus \mathscr{H}$ of the form

$$
L=\partial^{N}+u_{N-1} \partial^{N-1}+\cdots+u_{1} \partial+u_{0}+\Phi \partial^{-1} \Psi, \quad N=1,2, \ldots
$$

Following the proof of Theorem 1 we observe that in the chart $(l, \Phi, \Psi)$ the tensor (3.3) can be restricted to purely differential operators $l$. Observing

$$
\left[P_{\geqq 0}\left(\frac{\delta H}{\delta l}\right), l\right]-P_{\geqq 0}\left(\left[\frac{\delta H}{\delta l}, l\right]\right)=-\left[P_{<0}\left(\frac{\delta H}{\delta l}\right), l\right]+P_{<0}\left(\left[\frac{\delta H}{\delta l}, l\right]\right)
$$

it is easy to see that the image of $\tilde{\mathscr{P}}_{1}$ produces operators with highest differential orders being two orders less than the highest order of $l$. Hence, if we start with $l=u_{N} \partial^{N}+u_{N-1} \partial^{N-1}+$ lower orders, the two highest coefficients of $u_{N}$ and $u_{N-1}$ in $l$ will have no time evolution and may be chosen arbitrarily. We have chosen $u_{N}=1$ and $u_{N-1}=0$. After the shift $l \rightarrow L=l+\Phi \partial^{-1} \Psi$ the image (3.2) of the tensor (3.3) is tangent to the submanifold of operators (3.14).

With

$$
P_{\geqq 0}\left(L \frac{\delta H}{\delta L}\right) L-L P_{\geqq 0}\left(\frac{\delta H}{\delta L} L\right)=-P_{<0}\left(L \frac{\delta H}{\delta L}\right) L+L P_{<0}\left(\frac{\delta H}{\delta L} L\right)
$$

a similar highest order analysis applies to the quadratic tensor, too. For $L=u_{N} \partial^{N}+$ lower orders the highest order produced by $\mathscr{P}_{2}$ will be $N-1$. Hence we may choose $u_{N}$ arbitrarily, where our choice is $u_{N}=1$. A more sophisticated argument is needed to show that the negative differential orders of $L$ may be restricted to the particular form $\Phi \partial^{-1} \Psi$. For an arbitrary Hamiltonian $H$ we consider the associated Hamiltonian system

$$
\begin{aligned}
& \frac{d}{d t} L=P_{\geqq 0}\left(L \frac{\delta H}{\delta L}\right) L-L P_{\geqq 0}\left(\frac{\delta H}{\delta L} L\right)-\Phi \partial^{-1} \frac{\delta H}{\delta \Phi} L+L \frac{\delta H}{\delta \Psi} \partial^{-1} \Psi, \\
& \frac{d}{d t} \Phi=P_{0}\left(L \frac{\delta H}{\delta L} \Phi\right)-\Phi D^{-1}\left(\Phi \frac{\delta H}{\delta \Phi}\right)+\Phi D^{-1}\left(\Psi \frac{\delta H}{\delta \Psi}\right)+P_{0}\left(L \frac{\delta H}{\delta \Psi}\right), \\
& \frac{d}{d t} \Psi=-P_{0}\left(L^{*}\left(\frac{\delta H}{\delta L}\right)^{*} \Psi\right)+\Psi D^{-1}\left(\Phi \frac{\delta H}{\delta \Phi}\right)-P_{0}\left(L^{*} \frac{\delta H}{\delta \Phi}\right)-\Psi D^{-1}\left(\Psi \frac{\delta H}{\delta \Psi}\right),
\end{aligned}
$$

where $\frac{\delta H}{\delta L} \in g$ is a pseudo-differential operator and $\frac{\delta H}{\delta \Phi}, \frac{\delta H}{\delta \Psi} \in \mathscr{H}$ are functions, respectively. We have to show that these equations are consistent if we assume that the negative part of $L$ is given by $P_{<0}(L)=\Phi \partial^{-1} \Psi$. The negative part of the evolution of $L$ can be rewritten as

$$
\begin{aligned}
P_{<0}\left(\frac{d}{d t} L\right)= & P_{<0}\left(P_{\geqq 0}\left(L \frac{\delta H}{\delta L}\right) \Phi \partial^{-1} \Psi-\Phi \partial^{-1} \Psi P_{\geqq 0}\left(\frac{\delta H}{\delta L} L\right)\right. \\
& \left.-\Phi \partial^{-1} \frac{\delta H}{\delta \Phi} L+L \frac{\delta H}{\delta \Psi} \partial^{-1} \Psi\right) .
\end{aligned}
$$


We note that for any differential operator $B=P_{\geqq 0}(B)$ one has

$$
P_{<0}\left(B \partial^{-1}\right)=P_{0}(B) \partial^{-1}, \quad P_{<0}\left(\partial^{-1} B\right)=\partial^{-1} P_{0}\left(B^{*}\right),
$$

so that (3.21) yields

$$
\begin{aligned}
P_{<0}\left(\frac{d}{d t} L\right)= & P_{0}\left(L \frac{\delta H}{\delta L} \Phi\right) \partial^{-1} \Psi-\Phi \partial^{-1} P_{0}\left(L^{*}\left(\frac{\delta H}{\delta L}\right)^{*} \Psi\right)-\Phi \partial^{-1} P_{0}\left(L^{*} \frac{\delta H}{\delta \Phi}\right) \\
& +P_{0}\left(L \frac{\delta H}{\delta \Psi}\right) \partial^{-1} \Psi-\Phi \partial^{-1} \frac{\delta H}{\delta \Phi} \Phi \partial^{-1} \Psi+\Phi \partial^{-1} \Psi \frac{\delta H}{\delta \Psi} \partial^{-1} \Psi .
\end{aligned}
$$

Using the evolutions (3.19), (3.20) for the functions $\Phi$ and $\Psi$ one obtains

$$
\begin{aligned}
\frac{d}{d t}\left(P_{<0}(L)-\Phi \partial^{-1} \Psi\right)= & \Phi \partial^{-1}\left(\Psi \frac{\delta H}{\delta \Psi}-\frac{\delta H}{\delta \Phi} \Phi\right) \partial^{-1} \Psi \\
& -\left[D^{-1}\left(\Psi \frac{\delta H}{\delta \Psi}-\frac{\delta H}{\delta \Phi} \Phi\right), \Phi \partial^{-1} \Psi\right],
\end{aligned}
$$

where we have used $\frac{\delta H}{\delta \Phi} \Phi=\left[\partial, D^{-1}\left(\frac{\delta H}{\delta \Phi} \Phi\right)\right], \Psi \frac{\delta H}{\delta \Psi}=\left[\partial, D^{-1}\left(\Psi \frac{\delta H}{\delta \Psi}\right)\right]$. Observing

$$
\begin{aligned}
\Phi \partial^{-1} \alpha \partial^{-1} \Psi & =\Phi \partial^{-1}\left(D^{-1}(\alpha)\right)_{x} \partial^{-1} \Psi=\Phi \partial^{-1}\left(\partial\left(D^{-1}(\alpha)\right)-\left(D^{-1}(\alpha)\right) \partial\right) \partial^{-1} \Psi \\
& =\left[D^{-1}(\alpha), \Phi \partial^{-1} \Psi\right]
\end{aligned}
$$

for an arbitrary function $\alpha$ it is readily verified that (3.24) vanishes. This finishes the calculation showing that $\mathscr{P}_{2}$ can be properly restricted to (3.15).

As a result the equations (3.10) can all be restricted to the submanifold of operators (3.14), because the equations are Hamiltonian w.r.t. the extended linear bracket $\mathscr{P}_{1}$. The restriction of $\mathscr{P}_{1}$ yields a proper first Hamiltonian formulation for these restricted evolutions. However, the extended quadratic bracket $\mathscr{P}_{2}$ can be properly restricted only to the larger submanifolds (3.15). Hence, an additional constraint $u_{N-1}=0$ has to be imposed. Using Dirac reduction, it is always possible to perform a non-trivial reduction of $\mathscr{P}_{2}$ to the manifold of operators (3.14), so that both $\mathscr{P}_{1}$ as well as $\mathscr{P}_{2}$ give rise to Hamiltonian formulations of the equations (3.10) restricted to Lax operators of the form (3.14). A few simple examples will be worked out in Sect. 6.

Theorem 5. On operators of the form (3.14) the restriction of $\mathscr{P}_{1}$ coincides with the restriction of the linear bracket (3.1). On operators of the form (3.15) the restriction of $\mathscr{P}_{2}$ coincides with the restriction of the quadratic bracket (3.6).

Proof. We first observe that both (3.1) and (3.6) can indeed be properly restricted. Insertion of $L=\partial^{N}+$ lower orders into (3.1) and (3.6) in conjunction with (3.16) and (3.17) shows that for any covector field $\frac{\delta H}{\delta L} \in g$ the tensors (3.1) and (3.6) produce operators of highest order $N-2$ and $N-1$, respectively. This takes care of the special form $L=\partial^{N}+u_{N-2} \partial^{N-2}+$ lower orders in (3.14) and $L=\partial^{N}+u_{N-1} \partial^{N-1}+$ lower orders in (3.15), respectively. Considering the special 
form $L=l+\Phi \partial^{-1} \Psi$ with a purely differential operator $l=P_{\geqq 0}(l)$ we consider the negative part of (3.1) and (3.6) yielding

$$
\begin{aligned}
& P_{<0}\left(\left[P_{\geqq 0}\left(\frac{\delta H}{\delta L}\right), L\right]-P_{\geqq 0}\left(\left[\frac{\delta H}{\delta L}, L\right]\right)\right) \\
& =P_{<0}\left(\left[P_{\geqq 0}\left(\frac{\delta H}{\delta L}\right), \Phi \partial^{-1} \Psi\right]\right) \\
& =P_{0}\left(\frac{\delta H}{\delta L} \Phi\right) \partial^{-1} \Psi-\Phi \partial^{-1} P_{0}\left(\left(\frac{\delta H}{\delta L}\right)^{*} \Psi\right)
\end{aligned}
$$

and

$$
\begin{aligned}
P_{<0}( & \left.P_{\geqq 0}\left(L \frac{\delta H}{\delta L}\right) L-L P_{\geqq 0}\left(\frac{\delta H}{\delta L} L\right)\right) \\
= & P_{<0}\left(P_{\geqq 0}\left(L \frac{\delta H}{\delta L}\right) \Phi \partial^{-1} \Psi-\Phi \partial^{-1} \Psi P_{\geqq 0}\left(\frac{\delta H}{\delta L} L\right)\right) \\
& =P_{0}\left(L \frac{\delta H}{\delta L} \Phi\right) \partial^{-1} \Psi-\Phi \partial^{-1} P_{0}\left(L^{*}\left(\frac{\delta H}{\delta L}\right)^{*} \Psi\right)
\end{aligned}
$$

respectively. In both cases these expressions are tangent to $P_{<0}(L)=\Phi \partial^{-1} \Psi$ for arbitrary covector fields $\frac{\delta H}{\delta L} \in g$, so that the manifold $\left\{L=l+\Phi \partial^{-1} \Psi ; l=\right.$ purely differential\} is a proper Poisson submanifold for the tensors (3.1) and (3.6). We show that the restrictions coincide with the restrictions of $\mathscr{P}_{1}$ and $\mathscr{P}_{2}$. For this we define a dual parametrization for $L=l+\Phi \partial^{-1} \Psi$ by $\frac{\delta H}{\delta L}=\frac{\delta H}{\delta l}+A$, where $\frac{\delta H}{\delta l}=P_{<0}\left(\frac{\delta H}{\delta l}\right)$ has no differential part and $A=P_{\geqq 0}(A)$ is any differential operator satisfying

$$
P_{0}(A \Phi)=\frac{\delta H}{\delta \Psi}, \quad P_{0}\left(A^{*} \Psi\right)=\frac{\delta H}{\delta \Phi}
$$

with given functions $\frac{\delta H}{\delta \Phi}$ and $\frac{\delta H}{\delta \Psi}$. This parametrization will in fact convert the trace duality (2.5) to the duality (2.15), because

$$
\begin{aligned}
\left\langle\frac{d L}{d t}, \frac{\delta H}{\delta L}\right\rangle & =\operatorname{tr}\left(\left(\frac{d l}{d t}+\frac{d \Phi}{d t} \partial^{-1} \Psi+\Phi \partial^{-1} \frac{d \Psi}{d t}\right)\left(\frac{\delta H}{\delta l}+A\right)\right) \\
& =\operatorname{tr}\left(\frac{d l}{d t} \frac{\delta H}{\delta l}\right)+\operatorname{tr}\left(\frac{d \Phi}{d t} \partial^{-1} \Psi A\right)+\operatorname{tr}\left(\Phi \partial^{-1} \frac{d \Psi}{d t} A\right) \\
& =\operatorname{tr}\left(\frac{d l}{d t} \frac{\delta H}{\delta l}\right)+\int \frac{d \Phi}{d t} P_{0}\left(A^{*} \Psi\right) d x+\int \frac{d \Psi}{d t} P_{0}(A \Phi) d x \\
& =\operatorname{tr}\left(\frac{d l}{d t} \frac{\delta H}{\delta l}\right)+\int\left(\frac{d \Phi}{d t} \frac{\delta H}{\delta \Phi}+\frac{d \Psi}{d t} \frac{\delta H}{\delta \Psi}\right) d x
\end{aligned}
$$


Inserting $\frac{\delta H}{\delta L}=\frac{\delta H}{\delta l}+A$ into the linear bracket (3.1) yields

$$
\begin{aligned}
\frac{d}{d t} L= & \frac{d l}{d t}+\frac{d \Phi}{d t} \partial^{-1} \Psi+\Phi \partial^{-1} \frac{d \Psi}{d t} \\
= & {\left[P_{\geqq 0}\left(\frac{\delta H}{\delta l}+A\right), l+\Phi \partial^{-1} \Psi\right]-P_{\geqq 0}\left(\left[\frac{\delta H}{\delta l}+A, l+\Phi \partial^{-1} \Psi\right]\right) } \\
= & {[A, l]+\left[A, \Phi \partial^{-1} \Psi\right]-P_{\geqq 0}\left(\left[\frac{\delta H}{\delta l}, l\right]\right) } \\
& -P_{\geqq 0}([A, l])-P_{\geqq 0}\left(\left[A, \Phi \partial^{-1} \Psi\right]\right) \\
= & P_{\geqq 0}\left(\left[l, \frac{\delta H}{\delta l}\right]\right)+P_{<0}\left(\left[A, \Phi \partial^{-1} \Psi\right]\right) \\
= & P_{\geqq 0}\left(\left[l, \frac{\delta H}{\delta l}\right]\right)+P_{0}(A \Phi) \partial^{-1} \Psi-\Phi \partial^{-1} P_{0}\left(A^{*} \Psi\right) \\
= & P_{\geqq 0}\left(\left[l, \frac{\delta H}{\delta l}\right]\right)+\frac{\delta H}{\delta \Psi} \partial^{-1} \Phi-\Phi \partial^{-1} \frac{\delta H}{\delta \Phi},
\end{aligned}
$$

i.e.

$$
\frac{d}{d t} l=P_{\geqq 0}\left(\left[l, \frac{\delta H}{\delta l}\right]\right), \quad \frac{d}{d t} \Phi=\frac{\delta H}{\delta \Psi}, \quad \frac{d}{d t} \Psi=-\frac{\delta H}{\delta \Phi} .
$$

On the other hand, in the chart given by $(l, \Phi, \Psi)$ the Hamiltonian equation w.r.t. $\mathscr{P}_{1}$ is given by (3.3). Observing

$$
\left[P_{\geqq 0}\left(\frac{\delta H}{\delta l}\right), l\right]-P_{\geqq 0}\left(\left[\frac{\delta H}{\delta l}, l\right]\right)=-P_{\geqq 0}\left(\left[P_{<0}\left(\frac{\delta H}{\delta l}\right), l\right]\right)
$$

for $l=P_{\geqq 0}(l)$ this coincides with (3.31).

A similar analysis applies to the quadratic bracket (3.6). Inserting $\frac{\delta H}{\delta L}=\frac{\delta H}{\delta l}+A$ with $\frac{\delta H}{\delta l}=P_{<0}\left(\frac{\delta H}{\delta L}\right)$ and $A=P_{\geqq 0}\left(\frac{\delta H}{\delta L}\right)$ satisfying (3.28) into (3.6) produces

$$
\begin{aligned}
\frac{d}{d t} l= & P_{\geqq 0}\left(l \frac{\delta H}{\delta l}\right) l-l P_{\geqq 0}\left(\frac{\delta H}{\delta l} l\right)+P_{\geqq 0}\left(l \frac{\delta H}{\delta l} \Phi \partial^{-1} \Psi\right)-P_{\geqq 0}\left(\Phi \partial^{-1} \Psi \frac{\delta H}{\delta l} l\right) \\
& +P_{\geqq 0}\left(l \frac{\delta H}{\delta \Psi} \partial^{-1} \Psi\right)-P_{\geqq 0}\left(\Phi \partial^{-1} \frac{\delta H}{\delta \Phi} l\right), \\
\frac{d}{d t} \Phi= & P_{0}\left(l \frac{\delta H}{\delta l} \Phi\right)-\Phi D^{-1}\left(\Phi \frac{\delta H}{\delta \Phi}\right)+\Phi D^{-1}\left(\Psi \frac{\delta H}{\delta \Psi}\right)+P_{0}\left(l \frac{\delta H}{\delta \Psi}\right), \\
\frac{d}{d t} \Psi= & -P_{0}\left(l^{*}\left(\frac{\delta H}{\delta l}\right)^{*} \Psi\right)+\Psi D^{-1}\left(\Phi \frac{\delta H}{\delta \Phi}\right)-P_{0}\left(l^{*} \frac{\delta H}{\delta \Phi}\right)-\Psi D^{-1}\left(\Psi \frac{\delta H}{\delta \Psi}\right),
\end{aligned}
$$


as the Hamiltonian equation in the chart $(l, \Phi, \Psi)$. To compare this with the restriction of (3.7) one starts with $\mathscr{P}_{2}$ in the chart $(L, \Phi, \Psi)$. Considering the map $L \rightarrow l=L-\Phi \partial^{-1} \Psi$ it is a straightforward calculation to check that the pushout of (3.7) by this map yields (3.33) for the case, where $l$ is a purely differential operator. Thus it is shown that $\mathscr{P}_{1}$ and $\mathscr{P}_{2}$ reduce to the same tensors as the original brackets (3.1) and (3.6).

We finally show that the restriction of the flows (2.10) to operators (3.14) corresponds to a symmetry reduction of the KP hierarchy. We briefly review the construction of the KP hierarchy in terms of the pseudo-differential operator (1.2) (see [19] for a simple introduction). One simultaneously considers the hierarchy of equations (1.1) for the operator (1.2). This, in terms of the fields $U, U_{2}, U_{3}, \ldots$ parametrizing $L_{\mathrm{KP}}$ yields an infinite set of coupled nonlinear evolution equations. With $t_{2}=y$ one uses the equation with $n=2$ to express all "auxiliary" fields $U_{2}, U_{3}, \ldots$ in terms of the prime field $U$ and its $y$-derivatives. Eliminating these fields in the equations (1.1) with $n \geqq 3$ one obtains a hierarchy of evolution equations for the prime field $U$ only. For $n=3$ this is the KP equation itself, for $n>3$ one obtains the higher flows commuting with the KP equation. Introducing

$$
L=\left(L_{\mathrm{KP}}\right)^{N}=\partial^{N}+u_{N-2} \partial^{N-2}+\cdots+u_{0}+\partial^{-1} u_{-1}+\cdots,
$$

one obtains an invertible map between the infinite sets of parameters $\left(U, U_{2}, U_{3}, \ldots\right)$ and $\left(u_{N-2}, u_{N-3}, \ldots\right)$ in terms of differential relations, e.g.

$$
\begin{aligned}
& u_{N-2}=N U, \quad u_{N-3}=N U_{2}+\frac{N(N-1)}{2} U_{x}, \ldots, \\
& u_{N-j}=N U_{j-1}+F_{j}\left(U, U_{2}, \ldots, U_{j-2}\right),
\end{aligned}
$$

with differential expressions $F_{j}$ of the indicated arguments. Hence we may think of the KP hierarchy (1.1) in terms of the fields $\left(U, U_{2}, U_{3}, \ldots\right)$ or equivalently in terms of the fields $\left(u_{N-2}, u_{N-3}, \ldots\right)$. The underlying Lax equation in terms of $L$ is

$$
\frac{d}{d t_{n}} L_{\mathrm{KP}}=\left[P_{\geqq 0}\left(\left(L_{\mathrm{KP}}\right)^{n}\right), L_{\mathrm{KP}}\right] \leftrightarrow \frac{d}{d t_{n}} L=\left[P_{\geqq 0}\left(L^{n / N}\right), L\right], \quad n=1,2,3, \ldots
$$

Hence, the KP hierarchy is connected to the equations (2.6) with $L=\partial^{N}+$ lower orders using fractional powers of the form $q=n / N, n=1,2, \ldots$ The previous arguments have shown that (2.6) may be restricted to operators of the form (3.14). Hence, we have a generalization of the well-known reduction process in Sato's theory by imposing the constraint

$$
\left(L_{\mathrm{KP}}\right)^{N}=\text { purely differential }+\Phi \partial^{-1} \Psi
$$

on (3.34). We give an interpretation of this constraint in terms of the prime field $U$ of (1.2) satisfying the KP equation and its higher flows. Calculating the $N$-th flow for $L_{\mathrm{KP}}$ one obtains

$$
\frac{d}{d t_{n}} L_{\mathrm{KP}}=\left[P_{\geqq 0}\left(\left(L_{\mathrm{KP}}\right)^{N}\right), L_{\mathrm{KP}}\right]=-\left[P_{<0}\left(\left(L_{\mathrm{KP}}\right)^{N}\right), L_{\mathrm{KP}}\right]=-\left[\Phi \partial^{-1} \Psi, L_{\mathrm{KP}}\right] .
$$

Inserting $L_{\mathrm{KP}}=\partial+U \partial^{-1}+\cdots$ one extracts the first negative differential order from (3.38) leading to

$$
\frac{d U}{d t_{N}}=(\Phi \Psi)_{x}
$$


Hence, for Lax operators of the form (3.14), the bi-Hamiltonian evolution equations (2.10) with $q=n / N, n=1,2, \ldots$, may be regarded as the KP hierarchy subject to the constraint (3.39), where $\Phi$ and $\Psi$ are eigenfunctions and adjoint eigenfunctions of the KP hierarchy. This type of reduction was observed before in a series of papers ([7-11,20-22]), our derivation yields a systematic interpretation of the bi-Hamiltonian aspects of these flows.

\section{Constrained Modified KP Flows and their Bi-Hamiltonian Structure}

It was shown in [26-29] that the Lax equations (2.6) can be modified to Lax hierarchies

$$
\frac{d}{d t} L=\left[P_{\geqq 1}\left(L^{q}\right), L\right],
$$

which still commute for different choices of the (integer or fractional) power $q$ labeling the elements of the hierarchy. In [29] it was discussed that the Poisson tensor

$$
\Theta_{1}(L): \frac{\delta H}{\delta L} \rightarrow\left[P_{\geqq 1}\left(\frac{\delta H}{\delta L}\right), L\right]-P_{\geqq-1}\left(\left[\frac{\delta H}{\delta L}, L\right]\right)
$$

on $g$ provides the "first" Hamiltonian structure for the equations (4.1). Here (4.2) is the Lie-Poisson structure of a Lie bracket on $g$, which is associated with an algebra decomposition of $g$ into two Lie-subalgebras $g=\left\{\sum_{n \geqq 1} a_{n} \partial^{n}\right\} \oplus\left\{\sum_{n<1} a_{n} \partial^{n}\right\}$. For a detailed discussion in terms of classical $r$-matrices we refer to [29]. There also a second bracket was proposed, from which the "second" Hamiltonian structure for some classes of integrable equations encoded in (4.1) can be extracted using Dirac reduction. However, this bracket did not define an abstract Poisson bracket on $g$, as the underlying $r$-matrix is neither skew adjoint nor does its skew adjoint part satisfy appropriate conditions $([30,31])$. It turns out that the results on the lifted quadratic bracket (3.7) of the last section provide the answer to the proper quadratic bracket for the Lax equations (4.1).

Theorem 6. (The modified quadratic bracket). The tensor

$$
\begin{aligned}
\Theta_{2}(L): \frac{\delta H}{\delta L} \rightarrow & P_{\geqq 0}\left(L \frac{\delta H}{\delta L}\right) L-L P_{\geqq 0}\left(\frac{\delta H}{\delta L} L\right)-\left[P_{0}\left(L \frac{\delta H}{\delta L}\right), L\right] \\
& -P_{-1}\left(\left[\frac{\delta H}{\delta L}, L\right]\right) L+\left[D^{-1}\left(\operatorname{res}\left(\left[\frac{\delta H}{\delta L}, L\right]\right)\right), L\right]
\end{aligned}
$$

defines a Poisson bracket on $g$. The tensors $\Theta_{1}$ (given by (4.2)) and $\Theta_{2}$ are compatible.

The proof of the Poisson properties of $\Theta_{2}$ is given in the following section, where it is pointed out that (4.3) is the image of the Poisson tensor (3.13) under a gauge transformation. The compatibility of $\Theta_{1}$ and $\Theta_{2}$ follows from the standard argument that the shift $L \rightarrow \tilde{L}=L-1$ produces the image $\tilde{\Theta}_{2}(\tilde{L})=\Theta_{1}(\tilde{L})+\Theta_{2}(\tilde{L})$.

We point out that according to [24] the residue of a commutator lies in the image of the differential operator, so that $D^{-1}\left(\operatorname{res}\left(\left[\frac{\delta H}{\delta L}, L\right]\right)\right)$ is well-defined. 
This is reflected by the fact that, regarding $D^{-1}$ as the inverse of $D=d / d x$ modulo integration constants, these integration constants drop out of the commutator following the application of the integration operator.

The two brackets given by $\Theta_{1}$ and $\Theta_{2}$ provide the bi-Hamiltonian formulation for the Lax equations (4.1):

$$
\frac{d}{d t} L=\left[P_{\geqq 1}\left(L^{q}\right), L\right]=\left\{\begin{array}{l}
\Theta_{1}(L) L^{q}=\Theta_{1}(L) \operatorname{grad} \frac{1}{q+1} \operatorname{tr}\left(L^{q+1}\right), \\
\Theta_{2}(L) L^{q-1}=\Theta_{2}(L) \operatorname{grad} \frac{1}{q} \operatorname{tr}\left(L^{q}\right) .
\end{array}\right.
$$

Theorem 7. (Restrictions). Both Poisson brackets given by $\Theta_{1}$ and $\Theta_{2}$ can be properly restricted to operators of the form

$$
L=\partial^{N}+v_{N-1} \partial^{N-1}+\cdots+v_{1} \partial+v_{0}+\partial^{-1} \psi, \quad N=1,2, \ldots
$$

Proof. Replacing

$$
\left[P_{\geqq 1}\left(\frac{\delta H}{\delta L}\right), L\right]-P_{\geqq-1}\left(\left[\frac{\delta H}{\delta L}, L\right]\right)=-\left[P_{<1}\left(\frac{\delta H}{\delta L}\right), L\right]+P_{<-1}\left(\left[\frac{\delta H}{\delta L}, L\right]\right)
$$

in $\Theta_{1}$ shows that for $L=\partial^{N}+$ lower orders the linear tensor produces operators of highest order $N-1$. Using the analogue of (3.17) it is readily seen that this is also true for $\Theta_{2}$. We still need to show that the negative parts of the images of $\Theta_{1,2}$ consist only of their first negative order $P_{-1}(\cdot)$, if $P_{<0}(L)=P_{-1}(L)=\partial^{-1} \psi$. For the linear tensor $\Theta_{1}$ this is easily seen observing

$$
\begin{aligned}
P_{<0}\left(\left[P_{\geqq 1}\left(\frac{\delta H}{\delta L}\right), L\right]\right) & =P_{<0}\left(\left[P_{\geqq 1}\left(\frac{\delta H}{\delta L}\right), \partial^{-1} \psi\right]\right) \\
& =-P_{<0}\left(\partial^{-1} \psi P_{\geqq 1}\left(\frac{\delta H}{\delta L}\right)\right) \\
& =-\partial^{-1} P_{0}\left(\left(P_{\geqq 1}\left(\frac{\delta H}{\delta L}\right)\right)^{*} \psi\right) .
\end{aligned}
$$

For the quadratic tensor we put $\alpha=D^{-1}\left(\operatorname{res}\left(\left[\frac{\delta H}{\delta L}, L\right]\right)\right)$, so that $P_{-1}\left(\left[\frac{\delta H}{\delta L}, L\right]\right)=\partial^{-1} \alpha_{x}$. Hence, the last two terms of (4.3) yield

$$
\begin{gathered}
-P_{-1}\left(\left[\frac{\delta H}{\delta L}, L\right]\right) L+\left[D^{-1}\left(\operatorname{res}\left(\left[\frac{\delta H}{\delta L}, L\right]\right)\right), L\right] \\
=-\partial^{-1} \alpha_{x} L+[\alpha, L]=\partial^{-1} \alpha \partial L+L \alpha .
\end{gathered}
$$

The negative part of the expression is given by $P_{<0}\left(\partial^{-1} \alpha \partial L+L \alpha\right)$ $=\partial^{-1} P_{0}\left((\partial L)^{*} \alpha\right)+\partial^{-1} \psi \alpha$. We note that all operators of the form $\partial^{-1}$ "function" are tangent to the negative part $\partial^{-1} \psi$ of $L$. By similar arguments also the negative part of the remaining first 3 terms in (4.3) are shown to be of the form $\partial^{-1}$ "function". Thus we have proven the invariance of the space (4.5) relative to the Poisson brackets given by $\Theta_{1,2}$. 
Remark 2. As the equations (4.1) are Hamiltonian they can be restricted to the invariant subspace (4.5). In this situation the evolutions of the functions $v_{0}$ and $\psi$ are calculated easily. Using the abbreviation $B=P_{\geqq 1}\left(L^{q}\right)$ one obtains the lowest order in (4.1) as

$$
\frac{d}{d t} \partial^{-1} \psi=P_{-1}([B, L])=-P_{-1}\left(\partial^{-1} \psi B\right)=-\partial^{-1} P_{0}\left(B^{*} \psi\right)
$$

Hence $\psi$ satisfies the time evolution

$$
\frac{d}{d t} \psi=-P_{0}\left(B^{*} \psi\right)
$$

for adjoint eigenfunctions associated with the Lax hierarchy (4.1) (which explains our notation $\psi$ for the lowest order field in (4.5)). The time evolution for the field $v_{0}$ is obtained as

$$
\frac{d}{d t} v_{0}=P_{0}([B, L])=P_{0}\left(B v_{0}\right)+P_{0}\left(B \partial^{-1} \psi\right)-P_{0}\left(\partial^{-1} \psi B\right) .
$$

We introduce $\phi=v_{0}+D^{-1}(\psi)$ with the integration $D^{-1}$ given by (3.8). The evolution for $\phi$ is calculated as

$$
\begin{aligned}
\frac{d}{d t} \phi & =P_{0}\left(B\left(\phi-D^{-1}(\psi)\right)\right)+P_{0}\left(B \partial^{-1} \psi\right)-P_{0}\left(\partial^{-1} \psi B\right)-D^{-1}\left(P_{0}\left(B^{*} \psi\right)\right) \\
& =P_{0}(B \phi)-P_{0}\left(\partial^{-1} \psi B\right)-D^{-1}\left(P_{0}\left(B^{*} \psi\right)\right)
\end{aligned}
$$

as one has

$$
\begin{aligned}
P_{0}\left(B D^{-1}(\psi)\right) & =P_{0}\left(\sum_{n \geqq 1} b_{n} \partial^{n} D^{-1}(\psi)\right)=\sum_{n \geqq 1} b_{n} \frac{d^{n-1} \psi}{d x^{n-1}}=P_{0}\left(\sum_{n \geqq 1} b_{n} \partial^{n} \partial^{-1} \psi\right) \\
& =P_{0}\left(B \partial^{-1} \psi\right)
\end{aligned}
$$

for $B=\sum_{n \geqq 1} b_{n} \partial^{n}$. We further observe that

$$
\begin{gathered}
P_{0}\left(\partial^{-1} \psi B\right)=\sum_{n \geqq 1} P_{0}\left(\partial^{-1} \psi b_{n} \partial^{n}\right)=\sum_{n \geqq 1}(-1)^{n-1} \frac{d^{n-1}\left(\psi b_{n}\right)}{d x^{n-1}}, \\
P_{0}\left(B^{*} \psi\right)=\sum_{n \geqq 1} P_{0}\left((-1)^{n} \partial^{n} b_{n} \psi\right)=\sum_{n \geqq 1}(-1)^{n} \frac{d^{n}\left(b_{n} \psi\right)}{d x^{n}} .
\end{gathered}
$$

Hence we may identify $D^{-1}\left(P_{0}\left(B^{*} \psi\right)\right)=-P_{0}\left(\partial^{-1} \psi B\right)$, so that (4.12) leads to

$$
\frac{d}{d t} \phi=P_{0}(B \phi) \text {. }
$$

This is the evolution of the eigenfunctions associated with the Lax equations (4.1). Hence the coefficient $v_{0}=\phi-D^{-1}(\psi)$ in (4.5) is expressed in terms of eigenfunctions and adjoint eigenfunctions.

As a consequence, the evolution equation (4.10) for the lowest order $\psi$ in (4.5) is obtained as a linear operator acting on $\psi$. Hence it can be restricted to the trivial solution $\psi=0$. In this case the resulting equation (4.15) for $v_{0}=\phi$ is given by the linear operator $B$ acting on $\phi$, which can be restricted to trivial solutions 
$\phi=\lambda=$ const, as $B=P_{\geqq 1}\left(L^{q}\right)$ has no zero order term. Hence, as already pointed out in [29], the equations (4.1) can be further restricted to subspaces of the form

and

$$
L=\partial^{N}+v_{N-1} \partial^{N-1}+\cdots+v_{1} \partial+v_{0}, \quad N=1,2, \ldots
$$

$$
L=\partial^{N}+v_{N-1} \partial^{N-1}+\cdots+v_{1} \partial+\lambda, \quad N=1,2, \ldots,
$$

where $\lambda$ is an arbitrary constant parameter. However, neither of these smaller subspaces are Poisson subspaces relative to any of the brackets $\Theta_{1,2}$. In fact, it can be shown that for the case (4.16) no Dirac reduction of $\Theta_{1}$ and $\Theta_{2}$ subject to the constraint $\psi=0$ exists, as in the Poisson tensors the corresponding matrix entry for $\psi$ vanishes. Hence, the restriction of (4.1) to (4.16) yields equations which do not inherit a Hamiltonian structure from $\Theta_{1}$ or $\Theta_{2}$. However, using the combined constraint $\psi=0, v_{0}=\lambda$ for (4.17), Dirac reduction of both tensors $\Theta_{1}$ and $\Theta_{2}$ is possible. The resulting restricted equations inherit a reduced bi-Hamiltonian structure from $\Theta_{1}$ and $\Theta_{2}$. A few simple examples are worked out in Sect. 6.

We finally remark that the equations (4.1) restricted to $(4.5) /(4.16) /(4.17)$ are reductions of the modified KP-hierarchy. We briefly review the results of [28]. There is shown that the modified KP equation is connected with the Lax operator

$$
L_{\mathrm{mKP}}=\partial+V+V_{1} \partial^{-1}+V_{2} \partial^{-2}+\cdots .
$$

One simultaneously considers the hierarchy of equations

$$
\frac{d}{d t} L_{\mathrm{mKP}}=\left[P_{\geqq 1}\left(\left(L_{\mathrm{mKP}}\right)^{n}\right), L_{\mathrm{mKP}}\right], \quad n=1,2,3, \ldots,
$$

for the operator (4.18). In terms of the fields $V, V_{1}, V_{2}, \ldots$ parametrizing $L_{\mathrm{mKP}}$ the flows (4.19) yield an infinite set of coupled evolution equations. With $t_{2}=y$ one uses the equation with $n=2$ to express all "auxiliary" fields $V_{1}, V_{2}, \ldots$ in terms of the prime field $V$ and its $y$-derivatives. Eliminating these fields in the equations (4.19) with $n \geqq 3$ one obtains a hierarchy of evolution equations for the prime field $V$ only. For $n=3$ this is the modified KP equation

$$
4 \frac{d}{d t_{3}} V_{x}=\left(V_{x x x}+6 V^{2} V_{x}\right)_{x}+3 V_{y y}+6 V_{x} V_{y}+6 V_{x x} D^{-1}\left(V_{y}\right)
$$

itself, for $n>3$ one obtains the higher flows commuting with the modified KP equation.

In analogy to the discussion of the last section connecting the KP hierarchy to the equations (2.6) restricted to (3.14), one may impose the constraint

$$
\left(L_{\mathrm{mKP}}\right)^{N}=\partial^{N}+v_{N-1} \partial^{N-1}+\cdots+v_{0}+\partial^{-1} \psi
$$

on (4.18). We give an interpretation of this constraint in terms of the prime field $V$ of (4.18) satisfying the modified KP equation and its higher flows. Calculating the $N^{\text {th }}$ flow for $L_{\text {mKP }}$ one obtains

$$
\begin{aligned}
\frac{d}{d t_{N}} L_{\mathrm{mKP}} & =\left[P_{\geqq 1}\left(\left(L_{\mathrm{mKP}}\right)^{N}\right), L_{\mathrm{mKP}}\right]=-\left[P_{<1}\left(\left(L_{\mathrm{mKP}}\right)^{N}\right), L_{\mathrm{mKP}}\right] \\
& =-\left[v_{0}+\partial^{-1} \psi, L_{\mathrm{mKP}}\right] .
\end{aligned}
$$


Inserting $L_{\mathrm{mKP}}=\partial+V+V_{1} \partial^{-1}+\ldots$ one extracts the zero differential order from (4.22) leading to $\frac{d V}{d t_{N}}=v_{0 x}$. We have seen that according to the previous considerations the zero order coefficient $v_{0}$ is connected with eigenfunctions and adjoint eigenfunctions of the modified KP-hierarchy. Using $v_{0}=\phi-D^{-1}(\psi)$ one concludes that for Lax operators of the form (4.5) the bi-Hamiltonian evolution equations (4.4) with $q=n / N, n=1,2, \ldots$, may be regarded as the modified KP hierarchy subject to the constraint

$$
\frac{d V}{d t_{N}}=\phi_{x}-\psi
$$

Here $\phi$ and $\psi$ are eigenfunctions and adjoint eigenfunctions of the modified KP hierarchy satisfying

$$
\frac{d}{d t_{n}} \phi=P_{0}\left(B_{n} \phi\right), \quad \frac{d}{d t_{n}} \psi=-P_{0}\left(B_{n}^{*} \psi\right), \quad B_{n}=P_{\geqq 1}\left(\left(L_{\mathrm{mKP}}\right)^{n}\right), \quad n=1,2, \ldots
$$

\section{Miura Links and Gauge Transformations as Poisson Maps}

As discussed in $[28,29]$ gauge transformations of the underlying Lax operators provide the Miura link between the KP hierarchy and the modified KP hierarchy as well as their constrained flows. The new feature to be discussed here is the fact that now Hamiltonian interpretations of the flows involving the eigenfunctions and adjoint eigenfunctions are available. It turns out that the gauge transformations are indeed canonical in the sense that the bi-Hamiltonian structure of the (constrained) KP hierarchy is mapped to the bi-Hamiltonian structure of the (constrained) modified KP hierarchy.

Theorem 8. (Gauge transformations). The gauge transformation

$$
\text { G: }(L, \Phi, \Psi) \in g \oplus \mathscr{H} \oplus \mathscr{H} \rightarrow \tilde{L}=\Phi^{-1} L \Phi \in g
$$

maps the Poisson brackets given by $\mathscr{P}_{1}$ and $\mathscr{P}_{2}$ to the Poisson brackets given by $\Theta_{1}$ and $\Theta_{2}$, respectively.

We only indicate the calculations necessary for the proof. One has to show

$$
\begin{aligned}
& \Theta_{1}(\tilde{L})=G^{\prime} \mathscr{P}_{1}(L, \Phi, \Psi) G^{\prime \dagger}, \\
& \Theta_{2}(\tilde{L})=G^{\prime} \mathscr{P}_{2}(L, \Phi, \Psi) G^{\prime \dagger},
\end{aligned}
$$

where

$$
G^{\prime}:\left(\begin{array}{c}
A \\
\alpha \\
\beta
\end{array}\right) \rightarrow \Phi^{-1} A \Phi+\left[\tilde{L}, \Phi^{-1} \alpha\right], \quad G^{\dagger}: B \rightarrow\left(\begin{array}{c}
\Phi B \Phi^{-1} \\
\Phi^{-1} \operatorname{res}([B, \tilde{L}]) \\
0
\end{array}\right)
$$


are the linearized map $G^{\prime}$ and its transposed $G^{\prime \dagger}$, i.e. one has

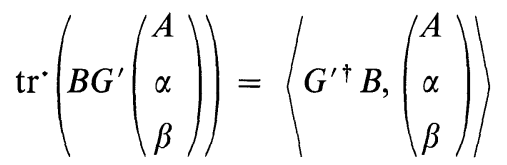

for all operators $A, B \in g$ and functions $\alpha, \beta \in \mathscr{H}$. Here the duality $\langle\cdot, \cdot\rangle$ is given by (2.15). Having identified $G^{\prime}$ and $G^{\prime \dagger}$ it now is a straightforward exercise to verify (5.2).

We remark that $\Theta_{1}$ and $\Theta_{2}$ may be understood as the images of the $(L, \Phi)$ blocks $\hat{\mathscr{P}}_{1}$ (3.12) and $\hat{\mathscr{P}}_{2}(3.13)$, respectively, as the gauge transformation does not involve the variable $\Psi$.

Observing $\operatorname{tr}\left(L^{q}\right)=\operatorname{tr}\left(\tilde{L^{q}}\right)$ we note that the Hamiltonians of the bi-Hamiltonian equations (3.10) are mapped to the Hamiltonians of the bi-Hamiltonian equations (4.4) (in the variable $\tilde{L}$ ). Hence, the Hamiltonian equations are connected via the gauge transformation:

$$
\left.\begin{array}{l}
\frac{d}{d t} L=\left[P_{\geqq 0}\left(L^{q}\right), L\right] \\
\frac{d}{d t} \Phi=P_{0}\left(L^{q} \Phi\right)
\end{array}\right\} \rightarrow \frac{d}{d t} \tilde{L}=\left[P_{\geqq 1}\left(\tilde{L^{q}}\right), \tilde{L}\right] .
$$

This - among other types of gauge transformations - was discussed in [28, 29]. There it was shown that Miura links can be extracted from these transformations. Here we have provided a Hamiltonian interpretation for the gauge transformation of Theorem 8.

We finally remark that the restrictions (3.14) naturally lead to the restrictions (4.5). Starting with $L$ of the form (3.14) the gauge transformed operator $\tilde{L}=\Phi^{-1} L \Phi$ has the form

$$
\tilde{L}=\partial^{N}+N \frac{\Phi_{x}}{\Phi} \partial^{N-1}+\cdots+v_{1} \partial+v_{0}+\partial^{-1} \Psi \Phi, \quad N=1,2, \ldots,
$$

where the highest field $v_{N-1}=N \frac{\Phi_{x}}{\Phi}$ is given in terms of $\Phi$ only. The other fields $v_{N-2}, \ldots, v_{0}$ are given as differential expression of $\Phi$ and the fields $u_{N-2}, \ldots, u_{0}$ parametrizing $L$. In particular, the zero order term is given by

$$
v_{0}=\Phi^{-1} P_{0}(L \Phi),
$$

the adjoint eigenfunction $\psi$ of (4.5) is given as the "squared eigenfunction" $\psi=\Psi \Phi$. The choice $\Psi=0$ leads to the additional restriction (4.16). In this case one can even impose the scattering equation (2.13) as a further constraint leading to $v_{0}=\lambda$, that is, to the restricted case (4.17).

\section{Examples}

6.1. Constrained KP Flows. We now work out a few examples of restrictions of the $\mathrm{KP}$ hierarchy connected with Lax operators of the form (3.14). We consider the evolution equations

$$
\frac{d}{d t_{n}} L=\left[P_{\geqq 0}\left(L^{n / N}\right), L\right], \quad L=\partial^{N}+u_{N-2} \partial^{N-2}+\cdots+u_{0}+\Phi \partial^{-1} \Psi
$$


using the fractional powers $n / N, n=1,2, \ldots$ The "basic $\operatorname{rot}^{\prime} L^{1 / N}=\partial+a_{0}$ $+a_{-1} \partial^{-1}+\cdots$ is calculated by requiring $\left(L^{1 / N}\right)^{N}=L$. This leads to a straightforward recursive scheme for the coefficients $a_{0}, a_{-1}, \ldots$ of $L^{1 / N}$, from which these coefficients can be calculated as differential expressions of $u_{N-2}, \ldots, u_{0}, \Phi, \Psi$. Higher fractional powers $L^{n / N}$ of $L$ are then calculated as powers $L^{n / N}=\left(L^{1 / N}\right)^{n}$ of this "basic root." By construction, the first equation with $n=1$ in the hierarchy (6.1) is given by $d L / d t_{1}=[\partial, L]=d L / d x$, so that the first time variable $t_{1}$ may be identified with the underlying space variable $x$. In order to compute the Poisson structures (3.2) and (3.7) we write $L=l+\Phi \partial^{-1} \Psi$ with $l=\partial^{N}+u_{N-2} \partial^{N-2}+\cdots+u_{0}$ (for (3.2)) and $l=\partial^{N}+u_{N-1} \partial^{N-1}+\cdots+u_{0}$ (for (3.7)), respectively. Regarding the functions $u_{0}, u_{1}, \ldots$ as coordinates parametrizing the operator $l$, the differential $\frac{\delta H}{\delta l}$ of a Hamiltonian function then is conveniently parametrized by

$$
\frac{\delta H}{\delta l}=\partial^{-1} \frac{\delta H}{\delta u_{0}}+\partial^{-2} \frac{\delta H}{\delta u_{1}}+\partial^{-3} \frac{\delta H}{\delta u_{2}}+\cdots
$$

with functions $\frac{\delta H}{\delta u_{i}}$. Now the trace duality $(2.5)$ becomes the usual duality bracket:

$$
\operatorname{tr}\left(\frac{d l}{d t} \frac{\delta H}{\delta l}\right)=\int \sum_{i} \frac{d u_{i}}{d t} \frac{\delta H}{\delta u_{i}} d x .
$$

$N=1$. For $L=\partial+\Phi \partial^{-1} \Psi$ the first nontrivial equations in (6.1) are given by

$$
\frac{d}{d t_{2}}\left(\begin{array}{c}
\Phi \\
\Psi
\end{array}\right)=\left(\begin{array}{l}
\Phi_{x x}+2 \Psi \Phi^{2} \\
\Psi_{x x}-2 \Phi \Psi^{2}
\end{array}\right), \quad \frac{d}{d t_{3}}\left(\begin{array}{l}
\Phi \\
\Psi
\end{array}\right)=\left(\begin{array}{c}
\Phi_{x x x}+6 \Psi \Phi \Phi_{x} \\
\Psi_{x x x}+6 \Phi \Psi \Psi_{x}
\end{array}\right),
$$

which are the first equations in the AKNS-hierarchy. Hamiltonian formulations for these equations are given by (3.10), where the first Hamiltonian functions are given by

$$
\operatorname{tr}(L)=\int \Phi \Psi d x, \frac{1}{2} \operatorname{tr}\left(L^{2}\right)=\frac{1}{2} \int\left(\Phi_{x} \Psi-\Phi \Psi_{x}\right) d x, \frac{1}{3} \operatorname{tr}\left(L^{3}\right)=\int\left(\Phi^{2} \Psi^{2}-\Phi_{x} \Psi_{x}\right) d x
$$

The linear bracket (3.2) is most conveniently calculated using (3.3) with $L=l+\Phi \partial^{-1} \Psi, l=\partial$. The $(l, l)$-block of the Poisson tensor (3.3) vanishes, so that the resulting Hamiltonian equation associated with a Hamiltonian function $H(\Phi, \Psi)$ is

$$
\frac{d}{d t}\left(\frac{\Phi}{\Psi}\right)=\left(\begin{array}{rr}
0 & 1 \\
-1 & 0
\end{array}\right)\left(\begin{array}{l}
\frac{\delta H}{\delta \Phi} \\
\frac{\delta H}{\delta \Psi}
\end{array}\right)
$$

For the quadratic Poisson bracket of the AKNS hierarchy we consider the reduction of (3.7) to the larger Poisson subspace given by (3.15), i.e. we first compute the quadratic bracket associated with operators of the form $L=\partial+u_{0}+\Phi \partial^{-1} \Psi=l+\Phi \partial^{-1} \Psi, l=\partial+u_{0}$. Inserting (6.2) into (3.33) the 
Hamiltonian equation associated with a Hamiltonian function $H\left(\Phi, \Psi, u_{0}\right)$ is calculated as

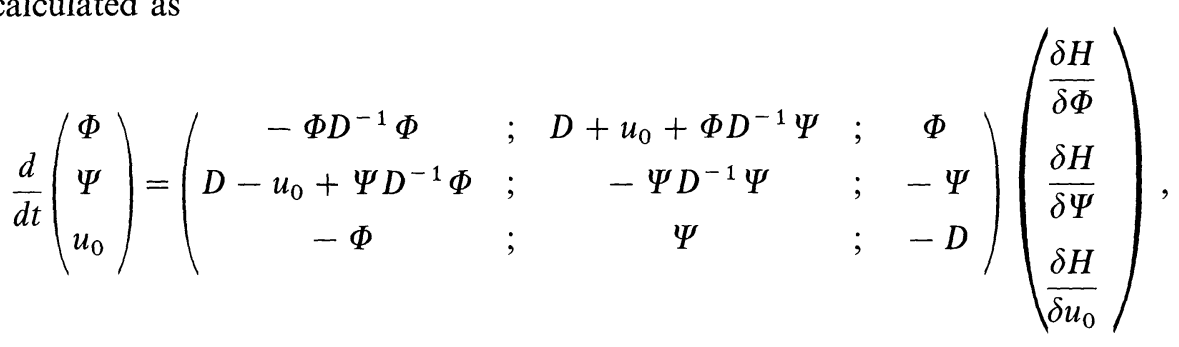

where $D=\frac{d}{d x}$, and $D^{-1}$ is given by (3.8). In order to obtain the second Poisson structure for the AKNS system we have to invoke Dirac reduction (see e.g. [31] for a description of this procedure in a suitable notation) to reduce (6.7) using the constraint $u_{0}=0$. The resulting Hamiltonian formulation of the constrained system is readily calculated as

$$
\frac{d}{d t}\left(\begin{array}{l}
\Phi \\
\Psi
\end{array}\right)=\left(\begin{array}{cc}
-2 \Phi D^{-1} \Phi & ; D+2 \Phi D^{-1} \Psi \\
D+2 \Psi D^{-1} \Phi ; & -2 \Psi D^{-1} \Psi
\end{array}\right)\left(\begin{array}{l}
\frac{\delta H}{\delta \Phi} \\
\frac{\delta H}{\delta \Psi}
\end{array}\right)
$$

which provides the second Hamiltonian formulation of the AKNS hierarchy.

$N=2$. For $L=\partial^{2}+u_{0}+\Phi \partial^{-1} \Psi$ the first nontrivial equations in (6.1) are given by

$$
\begin{aligned}
& \frac{d}{d t_{2}}\left(\begin{array}{c}
u_{0} \\
\Phi \\
\Psi
\end{array}\right)=\left(\begin{array}{c}
2(\Phi \Psi)_{x} \\
\Phi_{x x}+u_{0} \Phi \\
-\Psi_{x x}-u_{0} \Psi
\end{array}\right), \\
& 4 \frac{d}{d t_{3}}\left(\begin{array}{c}
u_{0} \\
\Phi \\
\Psi
\end{array}\right)=\left(\begin{array}{c}
u_{0 x x x}+6 u_{0} u_{0 x}+6 \Phi_{x x} \Psi-6 \Phi \Psi_{x x} \\
4 \Phi_{x x x}+6 u_{0} \Phi_{x}+3 u_{0 x} \Phi+6 \Psi \Phi^{2} \\
4 \Psi_{x x x}+6 u_{0} \Psi_{x}+3 u_{0 x} \Psi-6 \Phi \Psi^{2}
\end{array}\right),
\end{aligned}
$$

in accordance with the results of [9]. This represents the Korteweg-de Vries (KdV) hierarchy, coupled with its eigenfunctions and adjoint eigenfunctions ([13]). Hamiltonian formulations for these equations are given by (3.10), where the first Hamiltonian functions are given by

$$
\begin{aligned}
2 \operatorname{tr}\left(L^{1 / 2}\right) & =\int u_{0} d x, \quad \operatorname{tr}(L)=\int \Phi \Psi d x, \\
\frac{2}{3} \operatorname{tr}\left(L^{3 / 2}\right) & =\int\left(\frac{1}{4} u_{0}^{2}+\frac{1}{2}\left(\Phi_{x} \Psi-\Phi \Psi_{x}\right)\right) d x .
\end{aligned}
$$


The linear bracket (3.2) is calculated using (3.3) with $L=l+\Phi \partial^{-1} \Psi, l=\partial^{2}+u_{0}$. Inserting (6.2) into (3.3) the Hamiltonian equation associated with a Hamiltonian function $H\left(u_{0}, \Phi, \Psi\right)$ is computed as

$$
\frac{d}{d t}\left(\begin{array}{c}
u_{0} \\
\Phi \\
\Psi
\end{array}\right)=\left(\begin{array}{rrr}
2 D & 0 & 0 \\
0 & 0 & 1 \\
0 & -1 & 0
\end{array}\right)\left(\begin{array}{l}
\frac{\delta H}{\delta u_{0}} \\
\frac{\delta H}{\delta \Phi} \\
\frac{\delta H}{\delta \Psi}
\end{array}\right)
$$

For the quadratic Poisson bracket we consider the reduction of (3.7) to the larger Poisson subspace given by (3.15), i.e. we first compute the quadratic bracket associated with operators of the form $L=l+\Phi \partial^{-1} \Psi, l=\partial^{2}+u_{1} \partial+u_{0}$. Inserting (6.2) into (3.33) the Hamiltonian equation associated with a Hamiltonian $H\left(u_{0}, \Phi, \Psi, u_{1}\right)$ is calculated as

$\frac{d}{d t}\left(\begin{array}{c}u_{0} \\ \Phi \\ \Psi \\ u_{1}\end{array}\right)$

$$
=\left(\begin{array}{ccccccc}
\theta_{u_{0} u_{0}} & ; & * & ; & * & ; & * \\
D \Phi+u_{1} \Phi & ; & -\Phi D^{-1} \Phi & ; & * & ; & * \\
D \Psi+\Psi D-u_{1} \Psi & ; & -D^{2}+D u_{1}-u_{0}+\Psi D^{-1} \Phi & ; & -\Psi D^{-1} \Psi & * \\
D^{2}-D u_{1} & ; & -\Phi & ; & \Psi & ; & -2 D
\end{array}\right)
$$

$$
\times\left(\begin{array}{c}
\frac{\delta H}{\delta u_{0}} \\
\frac{\delta H}{\delta \Phi} \\
\frac{\delta H}{\delta \Psi} \\
\frac{\delta H}{\delta u_{1}}
\end{array}\right),
$$

where $\theta_{u_{0} u_{0}}=D^{3}+u_{1} D^{2}-D^{2} u_{1}+D\left(u_{0}-\frac{1}{2} u_{1}^{2}\right)+\left(u_{0}-\frac{1}{2} u_{1}^{2}\right) D$ and the remaining entries are given by skew-symmetry. In order to obtain the second Poisson structure for the equations (6.9) have to invoke Dirac reduction to reduce (6.12) 
using the constraint $u_{1}=0$. The resulting Hamiltonian formulation of the constrained system is readily calculated as

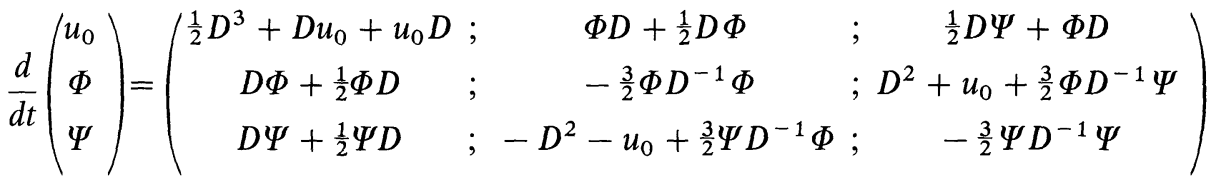

$$
\begin{aligned}
& \times\left(\begin{array}{l}
\frac{\delta H}{\delta u_{0}} \\
\frac{\delta H}{\delta \Phi} \\
\frac{\delta H}{\delta \Psi}
\end{array}\right) .
\end{aligned}
$$

This provides the second Hamiltonian formulation for the equations (6.9) $N=3$. For $L=\partial^{3}+u_{1} \partial+u_{0}+\Phi \partial^{-1} \Psi$ the first nontrivial equations in (6.1) are given by

$$
\begin{aligned}
3 \frac{d}{d t_{2}}\left(\begin{array}{c}
u_{1} \\
u_{0} \\
\Phi \\
\Psi
\end{array}\right) & =\left(\begin{array}{c}
-3 u_{1 x x}+6 u_{0 x} \\
\left(-2 u_{1 x x}+3 u_{0 x}-u_{1}^{2}+6 \Phi \Psi\right)_{x} \\
3 \Phi_{x x}+2 u_{1} \Phi \\
-3 \Psi_{x x}-2 u_{1} \Psi
\end{array}\right) \\
\frac{d}{d t_{3}}\left(\begin{array}{c}
u_{1} \\
u_{0} \\
\Phi \\
\Psi
\end{array}\right) & =\left(\begin{array}{c}
3(\Phi \Psi)_{x} \\
3\left(\Phi_{x} \Psi\right)_{x} \\
\Phi_{x x x}+u_{1} \Phi_{x}+u_{0} \Phi \\
\Psi_{x x x}+\left(u_{1} \Psi\right)_{x}-u_{0} \Psi
\end{array}\right)
\end{aligned}
$$

This is an extension of the Boussinesq system which essentially coincides with the system considered by Melnikov ([14]). We remark that the $t_{2}$-flow can be written in the more compact from

$$
\begin{aligned}
3 u_{1 t_{2} t_{2}}+\left(u_{1 x x}+2 u_{1}^{2}-12 \Phi \Psi\right)_{x x} & =0, \quad 3 \Phi_{t_{2}}-3 \Phi_{x x}-2 u_{1} \Phi=0, \\
3 \Psi_{t_{2}}+3 \Psi_{x x}+2 u_{1} \Psi & =0 .
\end{aligned}
$$

Hamiltonian formulations for these equations are given by (3.10), where the first Hamiltonian functions are given by

$$
\begin{gathered}
3 \operatorname{tr}\left(L^{1 / 3}\right)=\int u_{1} d x, \quad \frac{3}{2} \operatorname{tr}\left(L^{2 / 3}\right)=\int u_{0} d x, \quad \operatorname{tr}(L)=\int \Phi \Psi d x, \\
\frac{3}{4} \operatorname{tr}\left(L^{4 / 3}\right)=\int\left(\frac{1}{3} u_{1} u_{0}+\frac{1}{2}\left(\Phi_{x} \Psi-\Phi \Psi_{x}\right)\right) d x .
\end{gathered}
$$


The linear bracket (3.2) is calculated using (3.3) with $L=l+\Phi \partial^{-1} \Psi$, $l=\partial^{3}+u_{1} \partial+u_{0}$. Inserting (6.2) into (3.3) the Hamiltonian equation associated with a Hamiltonian function $H\left(u_{1}, u_{0}, \Phi, \Psi\right)$ is computed as

$$
\frac{d}{d t}\left(\begin{array}{c}
u_{1} \\
u_{0} \\
\Phi \\
\Psi
\end{array}\right)=\left(\begin{array}{cccc}
0 & 3 D & 0 & 0 \\
3 D & 0 & 0 & 0 \\
0 & 0 & 0 & 1 \\
0 & 0 & -1 & 0
\end{array}\right)\left(\begin{array}{c}
\frac{\delta H}{\delta u_{1}} \\
\frac{\delta H}{\delta u_{0}} \\
\frac{\delta H}{\delta \Phi} \\
\frac{\delta H}{\delta \Psi}
\end{array}\right)
$$

A second Poisson bracket can be computed with the restriction of (3.7) to the Poisson subspace given by operators of the form $L=\partial^{3}+u_{2} \partial^{2}+u_{1} \partial+u_{0}+$ $\Phi \partial^{-1} \Psi$. Proceeding as before, the restricted Poisson tensor may be computed by a straightforward, yet lengthy, calculation. Dirac reduction for the constraint $u_{3}=0$ provides the second Hamiltonian operator for the equations (6.14). We shall not display the resulting operator, as it is of a very complicated form.

We remark that according to the results of ([26]) each second of the Lax equations (6.1) (with odd values for $n$ ) can be restricted to Lax operators which are either symmetric or skew-symmetric. For the present case $N=3$ we may impose the constraint $u_{2}=0, u_{0}=\frac{1}{2} u_{1 x}$ and $\Psi=\Phi$, so that with $u_{1}=2 u$ the Lax operator $L=\partial^{3}+u \partial+\partial u+\Phi \partial^{-1} \Phi$ becomes skew-symmetric. In this case the $t_{3}$-evolution (6.14) reduces to

$$
\frac{d}{d t_{3}}\left(\begin{array}{l}
u \\
\Phi
\end{array}\right)=\left(\begin{array}{c}
3 \Phi \Phi_{x} \\
\Phi_{x x x}+2 u \Phi_{x}+u_{x} \Phi
\end{array}\right) .
$$

The $t_{5}$-flow in the hierarchy (6.1) associated with $L=\partial^{3}+u \partial+\partial u+\Phi \partial^{-1} \Phi$ is calculated as

$$
\begin{aligned}
9 u_{t_{5}}= & -u_{x x x x x}-10 u u_{x x x}-25 u_{x} u_{x x}-20 u^{2} u_{x}+30\left(u \Phi^{2}\right)_{x}+30 \Phi \Phi_{x x x}+45 \Phi_{x} \Phi_{x x} \\
9 \Phi_{t_{5}}= & 9 \Phi_{x x x x x}+30 u \Phi_{x x x}+45 u_{x} \Phi_{x x}+\left(35 u_{x x}+20 u^{2}+30 \Phi^{2}\right) \Phi_{x} \\
& +\left(10 u_{x x x}+20 u u_{x}\right) \Phi .
\end{aligned}
$$

This represents an extension of the Kupershmidt system ([32]).

6.2. Constrained Modified KP Flows. We now work out a few examples of restrictions of the modified KP hierarchy connected with Lax operators of the form (4.5). We consider the evolution equations

$$
\frac{d}{d t_{n}} L=\left[P_{\geqq 1}\left(L^{n / N}\right), L\right], L=\partial^{N}+v_{N-1} \partial^{N-1}+\cdots+v_{0}+\partial^{-1} \psi
$$

using the fractional powers $n / N, n=1,2, \ldots$ As in the case of the KP hierarchy, the "basic root" $L^{1 / N}=\partial+a_{0}+a_{-1} \partial^{-1}+\cdots$ is calculated by requiring $\left(L^{1 / N}\right)^{N}=L$. This leads to a straightforward recursive scheme for the coefficients $a_{0}, a_{-1}, \ldots$ of $L^{1 / N}$, from which these coefficients can be calculated as differential 
expressions of $v_{N-1}, \ldots, v_{0}, \psi$. Higher fractional powers $L^{n / N}$ of $L$ are then calculated as powers $L^{n / N}=\left(L^{1 / N}\right)^{n}$ of this "basic root." Again, the first equation with $n=1$ in the hierarchy (6.20) is given by $d L / d t_{1}=[\partial, L]=d L / d x$, so that the first time variable $t_{1}$ may be identified with the underlying space variable $x$. In order to compute the Poisson structures (4.2) and (4.3) we parametrize the differential of a Hamiltonian function $H$ by

$$
\frac{\delta H}{\delta L}=\frac{\delta H}{\delta \psi}+\partial^{-1} \frac{\delta H}{\delta v_{0}}+\partial^{-2} \frac{\delta H}{\delta v_{1}}+\partial^{-3} \frac{\delta H}{\delta v_{2}}+\cdots
$$

with functions $\frac{\delta H}{\delta v_{i}}$ and $\frac{\delta H}{\delta \psi}$. As a result, the trace duality (2.5) becomes the usual duality bracket:

$$
\operatorname{tr}\left(\frac{d L}{d t} \frac{\delta H}{\delta L}\right)=\int\left(\frac{d \psi}{d t} \frac{\delta H}{\delta \psi}+\sum_{i} \frac{d v_{i}}{d t} \frac{\delta H}{\delta v_{i}}\right) d x .
$$

$N=1$. For $L=\partial+v_{0}+\partial^{-1} \psi$ the first nontrivial equations in (6.20) are given by

$$
\frac{d}{d t_{2}}\left(\begin{array}{l}
v_{0} \\
\psi
\end{array}\right)=\left(\begin{array}{c}
\left(v_{0 x}+2 \psi+v_{0}^{2}\right)_{x} \\
\left(-\psi_{x}+2 v_{0} \psi\right)_{x}
\end{array}\right), \frac{d}{d t_{3}}\left(\begin{array}{c}
v_{0} \\
\psi
\end{array}\right)=\left(\begin{array}{c}
\left(v_{0 x x}+3 v_{0} v_{0 x}+v_{0}^{3}+6 v_{0} \psi\right)_{x} \\
\left(\psi_{x x}-3 v_{0} \psi_{x}+3 v_{0}^{2} \psi+3 \psi^{2}\right)_{x}
\end{array}\right)
$$

which are the first equations in the Kaup-Broer hierarchy discussed in [26]. Hamiltonian formulations for these equations are given by (4.4), where the first Hamiltonian functions are given by

$$
\operatorname{tr}(L)=\int \psi d x, \frac{1}{2} \operatorname{tr}\left(L^{2}\right)=\int v_{0} \psi d x, \quad \frac{1}{3} \operatorname{tr}\left(L^{3}\right)=\int\left(\psi^{2}-v_{0} \psi_{x}+v_{0}^{2} \psi\right) d x .
$$

Inserting (6.21) into (4.2) the Hamiltonian equation associated with a Hamiltonian function $H\left(v_{0}, \psi\right)$ is found to be

$$
\frac{d}{d t}\left(\begin{array}{l}
v_{0} \\
\psi
\end{array}\right)=\left(\begin{array}{ll}
0 & D \\
D & 0
\end{array}\right)\left(\begin{array}{l}
\frac{\delta H}{\delta v_{0}} \\
\frac{\delta H}{\delta \psi}
\end{array}\right)
$$

which represents the first Hamiltonian formulation for the flows (6.23). For the quadratic Poisson bracket we insert (6.21) into (4.3). By a straightforward computation the resulting Hamiltonian equation is calculated as

$$
\frac{d}{d t}\left(\begin{array}{l}
v_{0} \\
\psi
\end{array}\right)=\left(\begin{array}{cc}
2 D & ; D^{2}+D v_{0} \\
-D^{2}+v_{0} D & ; D \psi+\psi D
\end{array}\right)\left(\begin{array}{l}
\frac{\delta H}{\delta v_{0}} \\
\frac{\delta H}{\delta \psi}
\end{array}\right)
$$

which is the second Hamiltonian formulation of the Kaup-Broer hierarchy in accordance with [26].

Following Sect. 5 one can construct the Miura transformation between the AKNS hierarchy (6.4) and the Kaup-Broer hierarchy (6.23). One starts with the 
operator $L=\partial+\Phi \partial^{-1} \Psi$ associated with the AKNS hierarchy and performs the gauge transformation

$$
L \rightarrow \tilde{L}:=\frac{1}{\Phi} L \Phi=\partial+\frac{\Phi_{x}}{\Phi}+\partial^{-1} \Psi \Phi
$$

According to (5.5) the operator $\tilde{L}$ satisfies $d \tilde{L} / d t_{n}=\left[P_{\geqq 1}\left(\tilde{L}^{n}\right), \tilde{L}\right]$, if $\Phi$ and $\Psi$ solve the AKNS hierarchy. Hence, comparing $\tilde{L}$ and the operator $\partial+v_{0}+\partial^{-1} \psi$ associated with the Kaup-Broer hierarchy (6.23), we conclude that

$$
v_{0}=\frac{\Phi_{x}}{\Phi}, \quad \psi=\Phi \Psi
$$

provides the Miura-transformation between the AKNS hierarchy (6.4) and the Kaup-Broer hierarchy (6.23). Theorem 8 guarantees that the first Hamiltonian structure (6.6) of the AKNS hierarchy is Miura-related to the first Hamiltonian structure (6.25) of the Kaup-Broer hierarchy. The Miura-link between the second Hamiltonian structures (6.8) and (6.26) is less obvious, as Dirac reduction was used to obtain (6.8) for the general quadratic bracket $\Theta_{2}$ given by (4.3). However, a direct verification shows that also (6.8) and (6.26) are indeed linked via the Miura transformation (6.28).

$N=2$. For $L=\partial^{2}+v_{1} \partial+v_{0}+\partial^{-1} \psi$ the first nontrivial equations in (6.20) are given by

$$
\begin{aligned}
& \frac{d}{d t_{2}}\left(\begin{array}{l}
v_{1} \\
v_{0} \\
\psi
\end{array}\right)=\left(\begin{array}{c}
2 v_{0 x} \\
v_{0 x x}+v_{1} v_{0 x}+2 \psi_{x} \\
-\psi_{x x}+\left(v_{1} \psi\right)_{x}
\end{array}\right) \\
& 8 \frac{d}{d t_{3}}\left(\begin{array}{l}
v_{1} \\
v_{0} \\
\psi
\end{array}\right) \\
&=\left(\begin{array}{c}
2 v_{1 x x x}-3 v_{1}^{2} v_{1 x}+12\left(v_{0} v_{1}\right)_{x}+12 v_{0 x x}+24 \psi_{x} \\
8 v_{0 x x x}+12 v_{1} v_{0 x x}+6 v_{1 x} v_{0 x}+12 v_{1 x} \psi+24 v_{1} \psi_{x}+12 v_{0} v_{0 x}+3 v_{1}^{2} v_{0 x} \\
8 \psi_{x x x}-12 v_{1} \psi_{x x}-18 v_{1 x} \psi_{x}-6 v_{1 x x} \psi+12\left(v_{0} \psi\right)_{x}+3\left(v_{1}^{2} \psi\right)_{x}
\end{array}\right) .
\end{aligned}
$$

This represents the modified $\mathrm{KdV}$ hierarchy coupled with its eigenfunctions and adjoint eigenfunctions. Hamiltonian formulations for these equations are given by (4.4), where the first Hamiltonian functions are given by

$$
2 \operatorname{tr}\left(L^{1 / 2}\right)=\int\left(v_{0}-\frac{1}{4} v_{1}^{2}\right) d x, \quad \operatorname{tr}(L)=\int \psi d x
$$


Inserting (6.21) into (4.2) the Hamiltonian equation associated with a Hamiltonian function $H\left(v_{1}, v_{0}, \psi\right)$ is calculated as

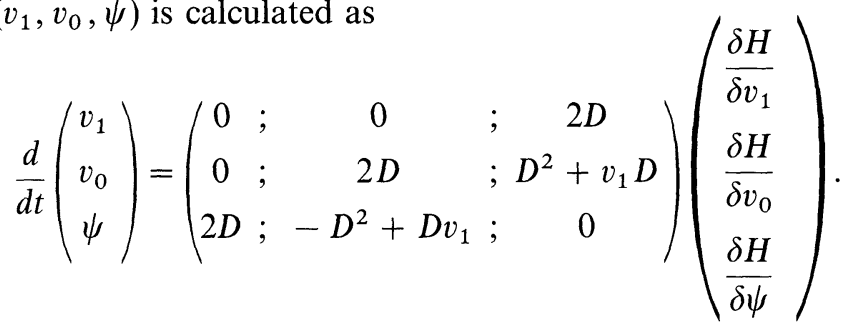

This provides the first Hamiltonian formulation for the equations (6.29). For the quadratic Poisson bracket we insert (6.21) into (4.3). The resulting Hamiltonian equation associated with a Hamiltonian function $H\left(v_{1}, v_{0}, \psi\right)$ is calculated as

$$
\begin{aligned}
& \frac{d}{d t}\left(\begin{array}{l}
v_{1} \\
v_{0} \\
\psi
\end{array}\right) \\
& =\left(\begin{array}{cccc}
6 D & ; & * & ; \\
4 v_{1} D & ; & * D^{3}+2 v_{1} D v_{1}+D v_{0}+v_{0} D & ; \\
2 D^{3}-2 D v_{1} D+2 v_{0} D & ; & \theta_{\psi v_{0}} & ;
\end{array}\right)\left(\begin{array}{l}
\frac{\delta H}{\delta v_{1}} \\
\frac{\delta H}{\delta v_{0}} \\
\frac{\delta H}{\delta \psi}
\end{array}\right) .
\end{aligned}
$$

with $\theta_{\psi v_{0}}=-D^{4}+D^{3} v_{1}+D v_{1} D^{2}-D v_{1} D v_{1}-v_{0} D^{2}+v_{0} D v_{1}+2 \psi D+D \psi$ and $\theta_{\psi \psi}=D v_{1} \psi+v_{1} \psi D+\psi D^{2}-D^{2} \psi$. The remaining entries of this Poisson tensor are given by skew-symmetry. This provides the second Hamiltonian formulation for the equations (6.29).

Following Sect. 5 one can construct the Miura transformation between the extended KdV hierarchy (6.9) and the extended modified KdV hierarchy (6.29). One starts with the operator $L=\partial^{2}+u_{0}+\Phi \partial^{-1} \Psi$ associated with the extended $\mathrm{KdV}$ hierarchy and considers the gauge transformation

$$
L \rightarrow \tilde{L}:=\frac{1}{\Phi} L \Phi=\partial^{2}+2 \frac{\Phi_{x}}{\Phi} \partial+\frac{1}{\Phi}\left(\Phi_{x x}+u_{0} \Phi\right)+\partial^{-1} \Psi \Phi .
$$

According to (5.5) the operator $\tilde{L}$ satisfies $d \tilde{L} / d t_{n}=\left[P_{\geqq 1}\left(\tilde{L^{n / 2}}\right), \tilde{L}\right]$, if $u_{0}, \Phi$ and $\Psi$ solve the extended $\mathrm{KdV}$ hierarchy (6.9). Hence, comparing $\tilde{L}$ and the operator $\partial^{2}+v_{1} \partial+v_{0}+\partial^{-1} \psi$ associated with the extended modified KdV hierarchy (6.29), we conclude that

$$
v_{1}=2 \frac{\Phi_{x}}{\Phi}, \quad v_{1}=\frac{1}{\Phi}\left(\Phi_{x x}+u_{0} \Phi\right), \quad \psi=\Phi \Psi
$$

provides the Miura-transformation between the extended KdV hierarchy (6.9) and the extended modified KdV hierarchy (6.29). Theorem 8 guarantees that the first Hamiltonian structure (6.11) of the extended KdV hierarchy is Miura-related to the 
first Hamiltonian structure (6.31) of the modified KdV hierarchy. The Miura-link between the second Hamiltonian structures (6.13) and (6.32) is less obvious, as Dirac reduction was used to obtain (6.32) from the general quadratic bracket $\Theta_{2}$ given by (4.3). However, a direct verification shows that also (6.13) and (6.32) are indeed linked via the Miura transformation (6.34).

\section{Conclusions and Perspectives}

We have given a systematic Hamiltonian interpretation of the symmetry constraint (3.39) of the KP hierarchy as proposed in [7-12, 21, 22]. It is shown that these constraints actually arise as proper Hamiltonian restrictions of the two Hamiltonian structures (3.1) and (3.6) underlying the classical results of Gelfand and Dikii. As a consequence, bi-Hamiltonian structures and recursion operators can be calculated algorithmically for the restricted KP flows. Further, a natural Miura link to restricted modified KP flows is given by simple gauge transformations involving eigenfunctions.

The systematic foundation of the constrained KP flows as restrictions of the general KP hierarchy (1.1) via (3.37) implies natural generalizations. Thus, it is readily seen that the flows (2.6) cannot only be restricted to submanifolds of the form (3.14), but any number $m$ of pairs of eigenfunctions and adjoint eigenfunctions may be considered. In particular, these flows can be restricted to operators of the form

$$
L=\partial^{N}+u_{N-2} \partial^{N-2}+\cdots+u_{0}+\sum_{i=1}^{m} \Phi_{i} \partial^{-1} \Psi_{i}
$$

In fact, it is easily seen that such operators again parametrize proper Poisson submanifolds for both brackets (3.1) and (3.6). The dynamics (2.6) will automatically imply that each $\Phi_{i}$ and each $\Psi_{i}$ is an eigenfunction and an adjoint eigenfunction associated with $L$, respectively, i.e.

$$
\begin{aligned}
\frac{d}{d t} L & =\left[P_{\geqq 0}\left(L^{q}\right), L\right], \quad \frac{d}{d t} \Phi_{i}=P_{0}\left(L^{q} \Phi_{i}\right), \\
\frac{d}{d t} \Psi_{i} & =-P_{0}\left(\left(L^{q}\right)^{*} \Psi_{i}\right), \quad i=1, \ldots, m .
\end{aligned}
$$

Following the arguments of Sect. 3 the restrictions of (7.2) to Lax operators of the form (7.1) may be understood as symmetry constraints

$$
\frac{d U}{d t_{N}}=\sum_{i=1}^{m}\left(\Phi_{i} \Psi_{i}\right)_{x}
$$

where $U$ is the prime field of the KP hierarchy.

Starting with (7.1) and choosing the first eigenfunction $\Phi_{1}$, say, to perform the gauge transformation $\tilde{L}=\Phi_{1}^{-1} L \Phi_{1}$, one encounters a transformed operator of the form

$$
\tilde{L}=\partial^{N}+v_{N-1} \partial^{N-1}+\cdots+v_{0}+\partial^{-1} \psi_{1}+\sum_{i=2}^{m} \phi_{i} \partial^{-1} \psi_{i}
$$


with new eigenfunctions $\phi_{i}=\frac{1}{\Phi_{1}} \Phi_{i}$ and new adjoint eigenfunction $\psi_{i}=\Psi_{i} \Phi_{1}$ satisfying the dynamics

$$
\begin{aligned}
\frac{d}{d t} \tilde{L} & =\left[P_{\geqq 1}\left(\tilde{L^{q}}\right), \tilde{L}\right], \quad \frac{d}{d t} \phi_{i}=P_{0}\left(P_{\geqq 1}\left(\tilde{L}^{q}\right) \phi_{i}\right), \\
\frac{d}{d t} \psi_{i} & =-P_{0}\left(\left(P_{\geqq 1}\left(\tilde{L}^{q}\right)\right)^{*} \psi_{i}\right), \quad i=1, \ldots, m .
\end{aligned}
$$

In particular, the original eigenfunction $\Phi_{1}$ is transformed into the trivial new eigenfunction $\phi_{1}=1$. It is a straightforward exercise to lift the general brackets (4.2) and (4.3) for the modified equations to extended Poisson structures for (7.5).

These considerations do not only lead to a systematic Hamiltonian concept for the constrained KP/modified KP flows. In fact, the most important implication of the results presented here seems a deeper understanding of the Hamiltonian nature of the gauge transformations $L \rightarrow \tilde{L}=\Phi^{-1} L \Phi$. Indeed, we have shown how a general quadratic Poisson bracket (4.3) for the modified equations is obtained as the image of the extended Poisson structure (3.7) under this type of gauge transformations. It turned out that the quadratic bracket proposed in ([29]) for the equations associated with the modified KP hierarchy has to be modified to the bracket (4.3) derived here.

We point out that it should also be possible to obtain a proper quadratic Poisson bracket for further hierarchies of Lax equations given by $\frac{d}{d t} L=\left[P_{\geqq 2}\left(L^{q}\right), L\right]$. These hierarchies are discussed in detail in ([29]), they lead to Harry Dym type equations associated with the constrained modified KP flows via reciprocal transformations involving the independent space variable $x$. Extending the quadratic bracket (4.3) to include eigenfunctions and adjoint eigenfunctions, we expect the reciprocal links of $([28,29])$ to produce the proper quadratic bracket for this class of equations.

Further, it was shown in $([30,31])$ that apart from the two brackets (3.1) and (3.6) there also exists a cubic bracket for the equations (2.6). Also this bracket admits a restriction to operators with negative parts given in the form $\Phi \partial^{-1} \Psi$ (or sums of such terms). Hence, it should be possible to construct a lifting of this bracket to the extended space $g \oplus \mathscr{H} \oplus \mathscr{H}$, which after gauge transformations and reciprocal links should produce proper cubic Poisson brackets for the constrained modified KP hierarchy and their reciprocally linked "Dym typ" counterpart.

Finally, we indicate an important line of further investigation, which should yield deeper insight into the Hamiltonian nature of gauge transformations. In $([28,29])$ classes of gauge transformations are discussed, which describe invariances of the Lax equations. In particular, the classical Darboux transformation

$$
L \rightarrow \hat{L}=\Phi \partial \Phi^{-1} L \Phi \partial^{-1} \Phi^{-1}
$$

leaves the dynamics (2.6) invariant, if $\Phi$ is an eigenfunction for $L$, i.e.

$$
\left.\begin{array}{rl}
\frac{d}{d t} L & =\left[P_{\geqq 0}\left(L^{q}\right), L\right] \\
\frac{d}{d t} \Phi & =P_{0}\left(L^{q} \Phi\right)
\end{array}\right\} \rightarrow \frac{d}{d t} \hat{L}=\left[P_{\geqq 0}\left(\hat{L}^{q}\right), \hat{L}\right] .
$$


As we now have the Poisson structures (3.2) and (3.7) involving the eigenfunction $\Phi$, the Hamiltonian character of the gauge transformation (7.6) can be investigated. We observe that the submanifold (3.14) is invariant under this transformation, in particular one finds

$$
\begin{aligned}
L & =\partial^{N}+u_{N-2} \partial^{N-2}+\cdots+u_{0}+\Phi \partial^{-1} \Psi \\
\rightarrow \hat{L} & =\partial^{N}+\hat{u}_{N-2} \partial^{N-2}+\cdots+\hat{u}_{0}+\hat{\Phi} \partial^{-1} \hat{\Psi}
\end{aligned}
$$

with the new eigenfunction $\hat{\Phi}=\Psi \Phi^{2}+\Phi\left(\Phi^{-1} P_{0}(L \Phi)\right)_{x}$ and the new adjoint eigenfunction $\hat{\Psi}=\Phi^{-1}$. This suggests to consider "lifted Darboux transformations" of the type

$$
\left(\begin{array}{l}
L \\
\Phi \\
\Psi
\end{array}\right) \rightarrow\left(\begin{array}{c}
\Phi \partial \Phi^{-1} L \Phi \partial^{-1} \Phi^{-1} \\
\Psi \Phi^{2}+\Phi\left(\Phi^{-1} P_{0}(L \Phi)\right)_{x} \\
\Phi^{-1}
\end{array}\right)
$$

on the extended space $g \oplus \mathscr{H} \oplus \mathscr{H}$. A detailed analysis of the Hamiltonian nature of such transformations shall be given elsewhere.

\section{References}

1. Airault, H., Mckean, H., Moser, J.: Commun. Pure Appl. Math. 30, 95 (1977)

2. Choodnovsky, D.V., Choodnovsky, G.V.: Nuovo Cimento B 40, 339 (1977)

3. Novikov, S.P.: Funct. Anal. Appl. 8, 236 (1974)

4. Fuchssteiner, B., Oevel, G.: Rev. Math. Phys. 1, 415 (1989)

5. Cao, C.W.: Henan Science 2, 2 (1987); Science in China (Scientia Sinica) 33, 528 (1990)

6. Orlov, A.Y., Schulman, E.I.: Lett. Math. Phys. 12, 171 (1986)

7. Konopelchenko, B.G., Strampp, W.: Inverse Problems 7, L17 (1991)

8. Konopelchenko, B.G., Sidorenko, J., Strampp, W.: Phys. Lett. 157 A, 17 (1991)

9. Sidorenko, J., Strampp, W.: Inverse Problems 7, L37 (1991)

10. Cheng, Y., Li, Y.: Phys. Lett. 157 A, 22 (1991)

11. Zeng Yunbo.: J. Phys. A 24, L1065 (1991)

12. Dubrovin, B., Malanyuk, T., Krichever, I., Makhankov, V.: Sov. J. Part. Nuc. 19, 252 (1988)

13. Yajima, N., Oikawa, M.: Progr. Theor. Phys. 56, 1719 (1976)

14. Melnikov, V.K.: Phys. Lett. 118A, 22 (1986); Lett. Math. Phys. 7, 129 (1983); Commun. Math. Phys. 112, 639 (1987); Commun. Math. Phys. 120, 451 (1989)

15. Sato, M.: RIMS Kokyuroku (Kyoto Univ.) 439, 30 (1981)

16. Sato, M., Sato, Y.: In Nonlinear Partial Differential Equations in Applied Science, Fujita, H., Lax, P.D., Strang G. (eds.) Tokyo: Kinokuniya/North Holland 1983

17. Date, E., Jimbo, M., Kashiwara, M., Miwa, T.: In Nonlinear Integrable Systems - Classical Theory and Quantum Theory, Jimbo, M., Miwa, T. (eds.) Singapore: World Scientific 1983

18. Jimbo, M., Miwa, T.: Publ. RIMS, Kyoto Univ., 19, 943 (1983)

19. Ohta, Y., Satsuma, J., Takahashi, D., Tokihiro, T.: Progr. Theor. Phys. Suppl. 94, 210 (1988)

20. Orlov, A.Y.: Symmetries for Unifying Different Soliton Systems into a Single Hierarchy. Preprint IINS/Oce-04/03, Moscow (1991)

21. Bing Xu, Li, Y.: J. Phys. A: Math. Gen. 25, 2957 (1992); Bing Xu.: A Unified Approach to Recursion Operators of the Reduced 1 + 1-Dimensional Systems. Preprint, Hefei (1992)

22. Cheng, Y.: J. Math. Phys. 33, 3774 (1992)

23. Gelfand, I.M., Dikii, L.A.: Funct. Anal. Appl. 10, 259 (1976)

24. Adler, M.: Invent. Math. 50, 219 (1979)

25. Semenov-Tian-Shansky, M.A.: Funct. Anal. Appl. 17, 259 (1983)

26. Kupershmidt, B.A.: Commun. Math. Phys. 99, 51 (1985)

27. Kiso, K.: Progr. Theor. Phys. 83, 1108 (1990) 
28. Oevel, W., Rogers, C.: Gauge Transformations and Reciprocal Links in $2+1$ Dimensions, to appear in Rev. Math. Phys. (1993)

29. Konopelchenko, B.G., Oevel, W.: An $r$-Matrix Approach to Nonstandard Classes of Integrable Equations, to appear in Publ. RIMS, Kyoto Univ.

30. Li, L.C., Parmentier, S.: Commun. Math. Phys. 125, 545 (1989)

31. Oevel, W., Ragnisco, O.: Physica A 161, 181 (1990)

32. Fordy, A.P., Gibbons, J.: Phys. Lett. 75 A, 325 (1980)

Communicated by $\mathrm{H}$. Araki 
